\title{
BIFURCATION FROM A HETEROCLINIC SOLUTION IN DIFFERENTIAL DELAY EQUATIONS
}

BY

\author{
HANS - OTTO WALTHER
}

\begin{abstract}
We study a class of functional differential equations $\dot{x}(t)=a f(x(t-1))$ with periodic nonlinearity $f: \mathbf{R} \rightarrow \mathbf{R}, 0<f$ in $(A, 0)$ and $f<0$ in $(0, B), f(A)=f(0)$ $=f(B)=0$. Such equations describe a state variable on a circle with one attractive rest point (given by the argument $\xi=0$ of $f$ ) and with reaction lag $a$ to deviations. We prove that for a certain critical value $a=a_{0}$ there exists a heteroclinic solution going from the equilibrium solution $t \rightarrow A$ to the equilibrium $t \rightarrow B$. For $a-a_{0}>0$, this heteroclinic connection is destroyed, and periodic solutions of the second kind bifurcate. These correspond to periodic rotations on the circle.
\end{abstract}

Introduction. Consider a state variable on a circle with one attractive rest point, and with a delayed reaction to deviations. A simple differential equation for such a system is

$$
\dot{y}(s)=f(y(s-a)), \quad a>0,
$$

or equivalently

$$
\dot{x}(t)=a f(x(t-1))
$$

where the function $f: \mathbf{R} \rightarrow \mathbf{R}$ is periodic with minimal period $-A+B, A<0<B$, $f(A)=0=f(0)=f(B), 0<f$ in $(A, 0)$ and $f<0$ in $(0, B)$. We regard the delay $a$ in the original equation as a parameter to be varied.

There are many interesting and complicated phenomena of bifurcation, partly understood or still to be studied, which occur in the set of solutions with values in $(A, B)$-where $f$ satisfies the condition $\xi f(\xi)<0$ for $\xi \neq 0$ for negative feedback with respect to the zero solution. The aim of the present paper, however, is different.

Several years ago, T. Furumochi [5] proved that, under additional hypotheses on $f$, there exists a parameter interval $I$ such that, for all $a \in I$, equation (af) has a periodic solution of the second kind; that is a solution $x: \mathbf{R} \rightarrow \mathbf{R}$ which satisfies

$$
x(t)=x(t+p)-(-A+B) \text { for every } t \in \mathbf{R},
$$

with some minimal "period" $p>0$. This corresponds to a periodic rotation of the state variable on the circle. Such solutions cannot arise in local bifurcation from equilibria.

In the theory of O.D.E.'s, say for vector fields on a cylinder, creation and destruction of periodic solutions which wind around the cylinder are well known.

Received by the editors July 24, 1984.

1980 Mathematics Subject Classification. Primary 34K15.

Key words and phrases. Characteristic equation, saddle point property, heteroclinic solution, Poincaré map, periodic solution of the second kind. 
Loosely spoken, they are due to the fact that the one-parameter family considered crosses the set of vector fields with homoclinic orbits around the cylinder. We refer the reader to [1, Chapter VIII and 3, Chapter 10]. For an interesting method in $\mathbf{R}^{n}$, $n>2$, see Sil'nikov [10].

We prove in this paper that for suitable functions $f$ the same type of bifurcation exists for the functional differential equation ( $a f)$ : There is a parameter $a_{0}=a_{0}(f)$ with a heteroclinic solution $\bar{x}^{0}: \mathbf{R} \rightarrow \mathbf{R}$ of $\left(a_{0} f\right)$ in the (one-dimensional) unstable manifold of the equilibrium $t \rightarrow A, \lim _{t \rightarrow+\infty} \bar{x}^{0}(t)=B$. For $a-a_{0}>0$ small enough, the heteroclinic connection is destroyed, and in every neighbourhood of $\left(a_{0}, \bar{x}^{0} \mid[-1,0]\right)$ there exist pairs $(a, \phi)$ with $a>a_{0}$ so that $\phi$ is the initial condition for a periodic solution of the second kind of $(a f)$.

The precise result is stated in Theorem 2 at the end of $\$ 4$. The assumptions on $f$ are given at the beginning of $\S 2$. They are designed in such a way that the proofs for existence and destruction of the heteroclinic solution do not become too lengthy. The final section, $§ 5$, shows that equations discussed by Furumochi [5] satisfy our assumptions. They describe a phase-locked loop for the control of a high frequency generator. Generalizations of our approach on how to find heteroclinic solutions are very well possible.

Existence of heteroclinic solutions of retarded functional differential equations was also proved by Hale and Rybakowski [8]. An entirely different idea, applying to a local problem of this type, is sketched in a report of Chow and Mallet-Paret [4]. For proofs possibly related to this idea see a paper in preparation of Mallet-Paret and Nussbaum [9].

The bifurcating periodic solutions of the second kind are obtained from a fixed point argument. For parameters $a$ close to $a_{0}$ we construct a self-mapping $P_{a}$ of a subset $D_{a}$ of a hyperplane transversal to the local unstable manifold of the equilibrium $t \rightarrow A$ so that fixed points define the desired periodic solutions. This construction requires information on solutions close to the equilibria given by $A$ and $B$. In our case these equilibria are hyperbolic. Estimates of the type we need were proved by Chafee [2], but for a slightly different situation with a center manifold at the critical parameter. We collect the analogous properties in Theorem 1 in $\S 3$. A proof will not be given since it is too close to Chafee's. But we choose notation similar to the one used in [2] in order to facilitate writing it down.

The crucial assumption for $P_{a}$ to become a self-mapping is that attraction to $B$ in the local stable manifold is stronger than repulsion in the local unstable manifold. The corresponding property of the characteristic equation for the linearization of (af) at $B$ should also imply that the periodic solutions which we obtain are stable and attractive. A proof would require more detailed knowledge on the local hyperbolic behaviour of the semiflow of $(a f)$. For reasons of length we have not attempted to include this in the present paper.

1. Preliminaries. Let $C$ and $C_{\mathbf{C}}$ denote the Banach spaces of continuous real and complex functions on the interval $[-1,0]$, with supremum-norm. For the constant function on $[-1,0]$ with value $\zeta$, we shall sometimes write $\phi_{\zeta} . B(\delta)$ stands for the closed ball of radius $\delta>0$ in the space $C$. 
Define $x_{t}$ by $x_{t}(\theta):=x(t+\theta)$ for $\theta \in[-1,0]$ whenever $x$ is a real or complex function on some set $D \subset \mathbf{R}$ with $[t-1,1] \subset D, t \in \mathbf{R}$. Let a continuous function $g$ : $\mathbf{R} \rightarrow \mathbf{R}$ be given. Let $a>0$. A solution of the functional differential equation

$$
\dot{x}(t)=a g(x(t-1))
$$

is either a differentiable function $x: \mathbf{R} \rightarrow \mathbf{R}$ which satisfies $(a g)$ everywhere, or a continuous function $x:[-1, \infty) \rightarrow \mathbf{R}$ which is differentiable on $\mathbf{R}^{+}:=(0, \infty)$ and satisfies (ag) for every $t>0$. In the linear case $g=\mathrm{id}$, complex-valued solutions of equation

$$
\dot{x}(t)=a x(t-1)
$$

are defined in the same way.

For every $\phi \in C, a$ and $g$ as above, there is a unique solution $x=x(\phi, a, g)$ : $[-1, \infty) \rightarrow \mathbf{R}$ of $(a g)$ with $x_{0}=\phi$. To prove this and the next statements one may use the formula

$$
x(t)=x(0)+a \int_{0}^{t} g(x(s-1)) d s=x(0)+a \int_{-1}^{t-1} g \circ \phi
$$

for $t \in[0,1]$, and iteration on the intervals $[n, n+1], n \in \mathbf{N}$.

We have continuous dependence on initial values and parameters: Let $T \geqslant 0$, $\phi \in C, a>0$ and $g$ be given. Then for every $\varepsilon>0$ there is $\delta>0$ with

$$
\sup _{t \in[-1, T]}\left|x(\phi, a, g)(t)-x\left(\phi^{\prime}, a^{\prime}, g\right)(t)\right| \leqslant \varepsilon
$$

for all $\phi^{\prime}$ and $a^{\prime}$ with $\left|\phi-\phi^{\prime}\right| \leqslant \delta$ and $\left|a-a^{\prime}\right| \leqslant \delta$.

Proposition 1. Suppose $g$ satisfies a Lipschitz condition and $A_{0} \subset \mathbf{R}^{+}$is bounded. Let $T \geqslant 0$. Then there exists a constant $k=k\left(A_{0}, g, T\right)$ with $\mid x_{T}(\phi, a, g)-$ $x_{T}\left(\phi^{\prime}, a, g\right)|\leqslant k| \phi-\phi^{\prime} \mid$ for all $\phi, \phi^{\prime} \in C$ and all $a \in A_{0}$.

We recall a few facts about the linear equation ( $a$ id) (see [6] for proofs). The relation $T_{a}(t, \phi)=x_{t}$ for $t \geqslant 0$ with the solution $x:[-1, \infty) \rightarrow \mathbf{C}$ of $(a \mathrm{id}), x_{0}=\phi$, defines a strongly continuous semigroup $T_{a}:[0, \infty) \times C_{\mathbf{C}} \rightarrow C_{\mathbf{C}}$ of bounded linear operators. The spectrum of the infinitesimal generator $G_{a}$ coincides with the zeros of the transcendental function $\Delta_{a}: \lambda \rightarrow \lambda-a e^{-\lambda}$. Every such zero $\lambda$ is an eigenvalue of $G_{a}$, and the algebraic multiplicity is equal to the order of $\lambda$ as a zero of $\Delta_{a}$.

Proposition 2. (i) For every $a>0$ there is precisely one positive zero $u(a)$ of $\Delta_{a}$. $u(a)$ is a simple zero.

(ii) The map $a \rightarrow u(a)$ is analytic and strictly increasing with $\lim _{a \rightarrow 0} u(a)=0$.

(iii) $\operatorname{Re} \lambda<\log a$ for every $a>0$ and for every zero $\lambda \neq u(a)$ of $\Delta_{a}$. In particular, $\lim _{a \rightarrow 0} \sup \left\{\operatorname{Re} \lambda \mid u(a) \neq \lambda \in \Delta_{a}^{-1}(0)\right\}=-\infty$.

Proof of (iii). Let $\Delta_{a}(\lambda)=0, \lambda \neq u(a)$. There are no real zeros except $u(a)$. Nonreal zeros occur in complex conjugate pairs. Therefore we may assume $\lambda=u+i v$ with $u \in \mathbf{R}, v>0$. Then $0<v=a e^{-u} \sin (-v)$. $\log a \leqslant u$ would imply $a e^{-u} \leqslant 1$, or $0<v \leqslant \sin (-v)=-\sin v$, a contradiction.

For proofs or methods on how to derive the results in the next part of this section the reader should consult e.g. [6 and 2]. We consider compact parameter intervals 
$A_{0} \subset \mathbf{R}^{+}$such that for every $a \in A_{0}$ there is no zero $\lambda$ of $\Delta_{a}$ with nonnegative real part except $u(a)$. Proposition 2 shows that $\max A_{0} \leqslant 1$ is sufficient for this.

It follows that for every $a \in A_{0}$ there is a decomposition $C=P_{a} \oplus Q_{a}$ into closed linear subspaces which are invariant with respect to the operators $T_{a}(t, \cdot)$, with $P_{a}=\mathbf{R} \Phi_{a}, \Phi_{a}(\theta)=e^{u(a) \theta}$ for $\theta \in[-1,0]$. The projection $p_{a}$ onto $P_{a}$ associated with the decomposition is explicitly given by $p_{a} \phi=\left(\Psi_{a}, \phi\right)_{a} \Phi_{a}$ for $\phi \in C$, where

$$
\left(\Psi_{a}, \phi\right)_{a}=\Psi_{a}(0) \phi(0)+a \int_{-1}^{0} \Psi_{a}(\theta+1) \phi(\theta) d \theta
$$

and

$$
\Psi_{a}(\theta)=\left[1+a e^{-u(a)}\right]^{-1} e^{-u(a) \theta} \text { for } \theta \in[0,1]
$$

Solutions with segments $x_{t}$ in $Q_{a}$ tend to zero as $t \rightarrow+\infty$, and nontrivial solutions with segments $x_{t}$ in $P_{a}$ increase exponentially. In fact, the latter are multiples of $t \rightarrow e^{u(a) t}$.

We come to the saddle point property for nonlinear equations. Suppose for simplicity that $g: \mathbf{R} \rightarrow \mathbf{R}$ is continuously differentiable, $g(\zeta)=0$ for some real number $\zeta$, and $g^{\prime}(\zeta)=1$. Then one can prove, for a parameter interval $A_{0}$ as above: There exist positive constants $\delta=\delta\left(A_{0}, g\right), \delta^{\prime}=\delta^{\prime}\left(A_{0}, g\right), L=L\left(A_{0}, g\right), K=$ $K\left(A_{0}, g\right), \gamma=\gamma\left(A_{0}, g\right)$ and two families of maps $u_{a g}: P_{a} \cap B(\delta) \rightarrow Q_{a}, s_{a g}$ : $Q_{a} \cap B(\delta) \rightarrow P_{a}, a \in A_{0}$, such that for every $a \in A_{0}$ the following holds true.

(i) $u_{a g}(0)=0, s_{a g}(0)=0$.

(ii) $u_{a g}$ and $s_{a g}$ satisfy a Lipschitz condition with Lipschitz constant $L$.

(iii) The graph of $u_{a g}$ is tangent to $P_{a}$ at $\phi=0$, the graph of $s_{a g}$ is tangent to $Q_{a}$ at $\phi=0$.

(iv) For every $\phi \in P_{a} \cap B(\delta)$ there exists a solution $x^{*}=x^{*}(\phi, a, g)$, defined on $\mathbf{R}$, of $(a g)$ with $x_{0}^{*}=\phi_{\zeta}+\phi+u_{a g}(\phi)$ and with

$$
\left|x_{t}^{*}-\phi_{\zeta}\right| \leqslant K e^{\gamma t}\left|\phi-\phi_{\zeta}\right| \text { for all } t \leqslant 0 \text {. }
$$

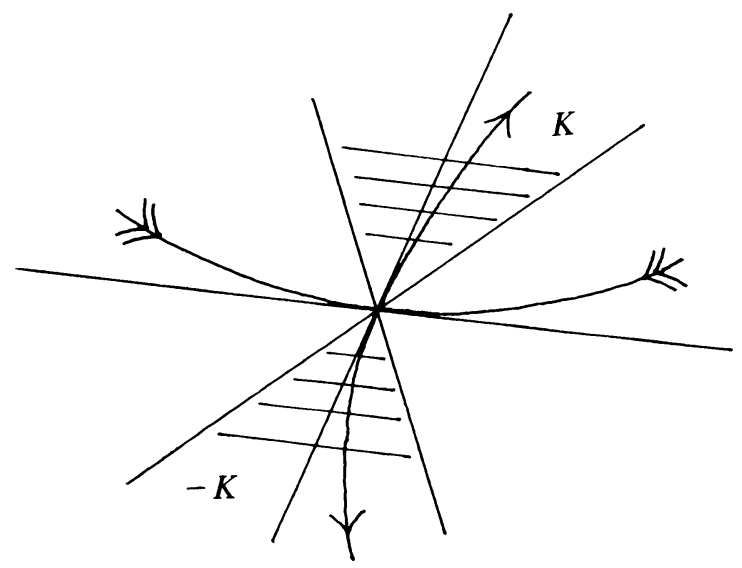

FIGURE 1 
(v) If $x: \mathbf{R} \rightarrow \mathbf{R}$ is any solution of (ag) with $\left|x_{t}-\phi_{\xi}\right| \leqslant \delta^{\prime}$ for all $t \leqslant 0$, then there exists $\phi \in P_{a} \cap B(\delta)$ with $x_{0}=\phi_{\zeta}+\phi+u_{a g}(\phi)$, and $x=x^{*}(\phi, a, g)$.

(vi) For every $\phi \in Q_{a} \cap B(\delta)$, the solution $x:[-1, \infty) \rightarrow \mathbf{R}$ of $(a g)$ with $x_{0}=\phi_{\zeta}$ $+\phi+s_{a g}(\phi)$ satisfies

$$
\left|x_{t}-\phi_{\zeta}\right| \leqslant K e^{-\gamma t}\left|\left(\phi_{\zeta}+\phi+s_{a g}(\phi)\right)-\phi_{\zeta}\right| \text { for all } t \geqslant 0 \text {. }
$$

(vii) If $x:[-1, \infty) \rightarrow \mathbf{R}$ is any solution of $(a g)$ with $\left|x_{t}-\phi_{\xi}\right| \leqslant \delta^{\prime}$ for all $t \geqslant 0$, then there exists $\phi \in Q_{a} \cap B(\delta)$ with $x_{0}=\phi_{\zeta}+\phi+s_{a g}(\phi)$.

Moreover, for every $\tilde{\delta} \in\left(-\delta^{\prime}, \delta^{\prime}\right)$, the map $A_{0} \ni a \rightarrow u_{a g}\left(\tilde{\delta} \Phi_{a}\right) \in C$ is continuous.

The sets $U_{a g}(\zeta):=\left\{\psi \in C \mid \psi=\phi_{\zeta}+\phi+u_{a g}(\phi)\right.$ for some $\left.\phi \in P_{a} \cap B(\delta)\right\}$ and $S_{a g}(\zeta):=\left\{\psi \in C \mid \psi=\phi_{\zeta}+\phi+s_{a g}(\phi)\right.$ for some $\left.\phi \in Q_{a} \cap B(\delta)\right\}$ are called local unstable and local stable manifolds of $\zeta$, or of $\phi_{\zeta}$.

Let $K$ denote the open convex cone of positive continuous functions in $C$. The representation of $p_{a}$ given above shows

Proposition 3. $p_{a} K \subset K$ for every $a \in A_{0}$.

Part of $U_{a g}(\zeta)$ is contained in $\phi_{\zeta}+(K \cup\{0\} \cup-K)$. This follows from tangency property (iii) above and from $P_{a} \subset K \cup\{0\} \cup-K$, but we do not need this here and the proof is omitted.

2. Equations with heteroclinic solutions. We specify a nonlinear function $f: \mathbf{R} \rightarrow \mathbf{R}$ and a parameter interval $A_{1}$ : Let a continuously differentiable function $\bar{f}: \mathbf{R} \rightarrow \mathbf{R}$ and real numbers $A<0, B>0, r>0, \xi_{1}, \xi_{2}$ be given with $A+2 r<\xi_{1}<\xi_{2}<-r$ $<r<B-r$ so that the following conditions are satisfied.

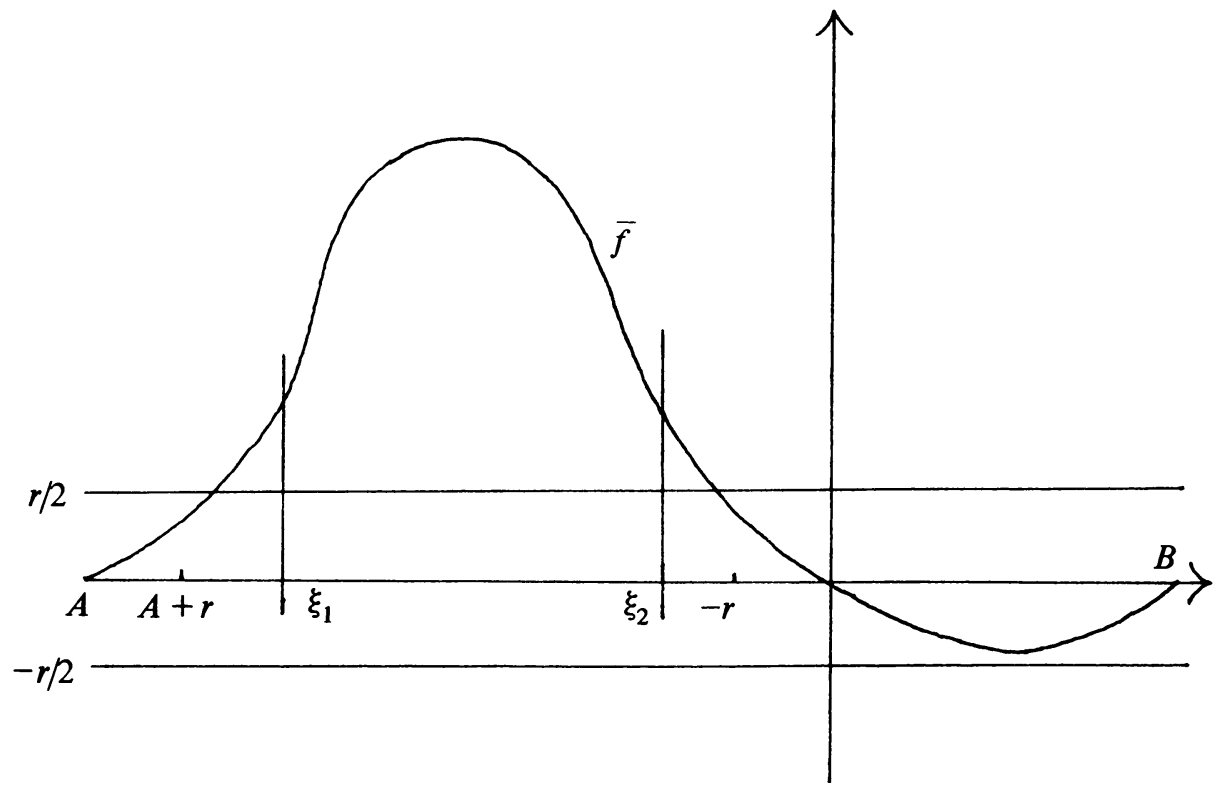

FiguRE 2 
(H1) $\bar{f}$ is periodic with minimal period $-A+B$.

(H2) $\bar{f}(A)=0,0<\bar{f}$ in $(A, 0), \bar{f}(0)=0, \bar{f}<0$ in $(0, B)$.

(H3) $|\bar{f}|<r / 2$ in $(A-r, A+r) \cup(-r, B-r]$.

(H4) There exists $q \in(0,1)$ with $|\bar{f}(\xi)| \leqslant q|\xi-A|$ for all $\xi \in(A-r, A+r)$.

(H5) $a^{+}:=\bar{f}^{\prime}(A)$ is positive with $\log a^{+}<-u\left(a^{+}\right)$, where $u=u\left(a^{+}\right)$satisfies $u=a^{+} e^{-u}$.

(H6) $\phi \in C, A \leqslant \phi \leqslant \xi_{1}, \phi(0)=\xi_{1}$ imply $\xi_{1}+\int \bar{f} \circ \phi \leqslant \xi_{2}$, and $\psi \in C, \xi_{1} \leqslant \psi \leqslant$ $\xi_{2}, \psi(0)=\xi_{2}$ imply $B+r<\xi_{2}+\int \bar{f} \circ \psi$.

We define $f(\xi):=\bar{f}(\xi) / a^{+}$so that $f^{\prime}(A)=1$. We choose $a^{-}$in $\left(0, a^{+}\right)$with $\left|a^{-} f\right|<r$ on all of $\mathbf{R}$, and we set $A_{1}:=\left[a^{-}, a^{+}\right]$.

Proposition 2 and $a^{-} \leqslant a \leqslant a^{+}$imply $\log a \leqslant \log a^{+}<-u\left(a^{+}\right) \leqslant-u(a)$. It follows that $A_{1}$ is a compact parameter interval in $\mathbf{R}^{+}$such that for every $a \in A_{1}$ there is no zero $\lambda$ of $\Delta_{a}$ with nonnegative real part except $u(a)$. Also, $f$ satisfies a Lipschitz condition. Therefore all the results of the preceding section apply to $g:=f$ and to $A_{1} . f$ and $A_{1}$ will be kept fixed from now on. We shall omit the argument $f$. For example, we write $x(\phi, a)$ and $u_{a}$ instead of $x(\phi, a, f)$ and $u_{a f}$, and we consider solutions of equation

$$
\dot{x}(t)=a f(x(t-1)) .
$$

Proposition 4. For $a \in A_{1}$ and for every solution $x: \mathbf{R} \rightarrow \mathbf{R}$ of equation (a) with $\lim _{t \rightarrow-\infty} x(t)=A$ and with $|x(t)-A| \leqslant r$ for all $t \leqslant 0$, either $x_{t} \in \phi_{A}+K$ on $(-\infty, 0]$, or $x_{t}=\phi_{A}$ on $(-\infty, 0]$, or $x_{t} \in \phi_{A}-K$ on $(-\infty, 0]$.

Proof. (a) The sign conditions on $f$, the uniqueness property for the initial value problem and the fact that equation $(a)$ is autonomous imply that in case $x_{t}=\phi_{A}$ $\left(x_{t} \in \phi_{A}+K\right)\left(\left(x_{t} \in \phi_{A}-K\right)\right)$ for some $t<0, x_{s}=\phi_{A}\left(x_{s} \in \phi_{A}+K\right)\left(\left(x_{s} \in \phi_{A}\right.\right.$ $-K))$ on $[t, 0]$.

(b) Let $x_{t}=\phi_{A}$ for some $t \leqslant 0$. Assume $x_{s} \neq \phi_{A}$ for some $s<t$. Then there exists $t^{*} \leqslant t-1$ with $x=A$ on $\left[t^{*}, 0\right]$ and $x\left(t^{*}+\theta\right) \neq A$ for some $\theta \in[-1,0)$. Hence $\dot{x}\left(t^{*}+\theta+1\right) \neq 0$ which leads to a contradiction.

(c) Let $x_{t} \neq \phi_{A}$ for some $t \leqslant 0$. (b) shows $x_{s} \neq \phi_{A}$ on $(-\infty, 0]$. In order to prove $x_{s} \in \phi_{A}+(K \cup-K)$ on $(-\infty, 0]$, assume $x_{s} \notin \phi_{A}+(K \cup-K)$ for some $s \leqslant 0$. Let $r^{\prime}:=\sup _{\sigma \leqslant s}\left|x_{\sigma}-\phi_{A}\right|=\sup _{\sigma \leqslant s}|x(\sigma)-A|$. Then $0<r^{\prime} \leqslant r$. Choose $s^{*} \leqslant s$ with $q r^{\prime}$ $<\left|x\left(s^{*}\right)-A\right| \leqslant r^{\prime}$. We have $0 \neq x_{s^{*}}-\phi_{A} \notin K \cup-K$ (otherwise $x_{s}-\phi_{A} \in K \cup-K$ because of (a)). Therefore $x\left(s^{*}+\theta\right)=A$ for some $\theta \in[-1,0]$, and

$$
x\left(s^{*}\right)=A+\int_{s^{*}+\theta}^{s^{*}} \dot{x}=A+\int_{s^{*}+\theta}^{s^{*}} a f(x(\sigma-1)) d \sigma .
$$

Using (H4) we find

$$
q r^{\prime}<\left|x\left(s^{*}\right)-A\right| \leqslant \int_{s^{*}+\theta}^{s^{*}} q|x(\sigma-1)-A| d \sigma \leqslant q r^{\prime},
$$

a contradiction.

(d) Altogether: $x_{t}=\phi_{A}$ for some $t \leqslant 0$ gives $x=A$ on $(-\infty, 0] \cdot x_{t} \neq \phi_{A}$ for some $t \leqslant 0$ gives $x_{s} \in \phi_{A}+(K \cup-K)$ on $(-\infty, 0]$ (see (c)). In case $x_{0} \in \phi_{A}+K$ there is no $s<0$ with $x_{s} \in \phi_{A}-K$ since this would imply $x_{0} \in \phi_{A}-K$ (see (a)). Therefore, 
$x_{s} \in \phi_{A}+K$ on $(-\infty, 0]$. In the same way we obtain $x_{s} \in \phi_{A}-K$ on $(-\infty, 0]$ if $x_{0} \in \phi_{A}-K$.

We choose positive constants $\delta_{1}=\delta\left(A_{1}, f\right), \delta_{2}=\delta^{\prime}\left(A_{1}, f\right), L_{1}=L\left(A_{1}, f\right), k_{1}=$ $K\left(A_{1}, f\right)$ and $\gamma_{1}=\gamma\left(A_{1}, f\right)$ such that the saddle point properties stated in $\$ 1$ hold true for $\zeta=A$, and moreover $\delta_{2}<\delta_{1}, k_{1} \geqslant 1$. Set

$$
\delta_{3}:=\left(2\left(L_{1}+1\right)\left(k_{1}+1\right)\right)^{-1} \min \left\{\delta_{2}, r\right\} \text {. }
$$

Choose $\delta \in\left(0, \delta_{3}\right)$. The map $A_{1} \ni a \rightarrow \phi_{a}:=\phi_{A}+\delta \Phi_{a}+u_{a}\left(\delta \Phi_{a}\right) \in C$ is continuous, with

$$
\left|\phi_{a}-\phi_{A}\right| \leqslant \delta+L_{1} \delta \leqslant \min \left\{\delta_{1}, \delta_{2}, r\right\} /\left(k_{1}+1\right) .
$$

(Recall $\left|\Phi_{a}\right|=1$.)

For $a \in A_{1}$, let $x^{a}: \mathbf{R} \rightarrow \mathbf{R}$ denote a solution of equation $(a)$ with $x_{0}^{a}=\phi_{a}$ and with $\left|x_{t}^{a}-\phi_{A}\right| \leqslant k_{1} e^{\gamma_{1} t}\left|\phi_{a}-\phi_{A}\right|$ for all $t \leqslant 0$. Clearly $\left|x_{t}^{a}-\phi_{A}\right|<\min \left\{r, \delta_{2}\right\}$ for all $t \leqslant 0$, and $\lim _{t \rightarrow-\infty} x^{a}(t)=A$.

Proposition 5. $x_{t}^{a} \in \phi_{A}+K$ on $(-\infty, 0]$, i.e. $x^{a}>A$ on $(-\infty, 0]$.

Proof. $p_{a}\left(x_{0}^{a}-\phi_{A}\right)=\delta \Phi_{a} \neq 0$ yields $x_{0}^{a} \neq \phi_{A}$. Proposition 4 now shows $x_{t}^{a} \in \phi_{A}$ $+K$ on $(-\infty, 0]$, or $x_{t}^{a} \in \phi_{A}-K$ on $(-\infty, 0]$. $x_{0}^{a} \in \phi_{A}-K$ would imply $\delta \Phi_{a}=$ $p_{a}\left(x_{0}^{a}-\phi_{A}\right) \in-K$ (see Proposition 3$)$, a contradiction.

Note that the last argument also justifies a part of Figure 1: It proves that the set $\left\{\phi \in U_{a}(A) \mid \phi=\phi_{A}+\psi+u_{a}(\psi)\right.$ with $\psi \in P_{a} \cap K$ and $\left.|\psi|<\delta_{3}\right\}$ is contained in $\phi_{A}+K$. In $\S 4$ we shall make use of

REMARK 1. $x_{t}^{a} \in U_{a}(A)$ for every $t \leqslant 0$.

Proof. For every $t \leqslant 0$, there exists a solution $\tilde{x}: \mathbf{R} \rightarrow \mathbf{R}$ of equation $(a)$ with $\tilde{x}_{0}=x_{t}^{a}$ and $|\tilde{x}| \leqslant \delta_{2}=\delta^{\prime}\left(A_{1}, f\right)$ on $(-\infty, 0]$-namely, $\tilde{x}(s)=x^{a}(s+t)$ for all $s \in \mathbf{R}$.

We want to single out a set $H \subset A_{1}$ such that $\lim _{t \rightarrow \infty} x^{a}(t)=B$ for all $a \in H$. We define $A^{+}:=\left\{a \in A_{1} \mid x_{t}^{a}>B\right.$ for some $\left.t>0\right\}, A^{-}:=\left\{a \in A_{1} \mid-r / 2<x_{t}^{a}<B-\right.$ $r / 2$ for some $t>0\} . A^{+}$and $A^{-}$are open subsets of $A_{1}$. The next results show that both are nonempty.

Proposition 6. (i) For every $a \in A_{1}$ there exists $t(a)>0$ with $A<x^{a}<-r / 2$ in $(-\infty, t(a))$ and $x^{a}(t(a))=-r / 2$. We have $\dot{x}^{a}>0$ in $(-\infty, t(a)+1]$.

(ii) $x^{a^{-}} \leqslant r$ in $[0, \infty)$.

(iii) There exists $t>0$ with $x^{a^{+}}>B$ on $[t-1, t]$.

Proof. (i) Let $a \in A_{1}$. We have $\phi_{a} \in \phi_{A}+K$ and $\left|\phi_{a}-\phi_{A}\right|<r$, and $A<x^{a}<A$ $+r$ on $(-\infty, 0] . A<\phi_{a}<A+r$ and equation $(a)$ show that $x^{a}$ is increasing on $[0, \infty)$ until it reaches the value $-r / 2$ at some time $t(a)>0$. On $[t(a)-1, t(a)]$, $A<x^{a}(s) \leqslant-r / 2$. Hence $\dot{x}^{a}>0$ on $[t(a), t(a)+1]$.

(ii) Set $x:=x^{a^{-}}$. Then $x_{0}<A+r<r$. Suppose there exists $s>0$ with $x(s)=r$ and $x<r$ on $[-1, s)$. It follows that $\dot{x}(s) \geqslant 0 .\left|a^{-} f\right|<r$ implies $|\dot{x}|<r$. Therefore $0 \leqslant x$ on $[s-1, s]$.

Case I: $0<x(s-1)$. Then $\dot{x}(s)<0$, a contradiction.

Case II: $x(s-1)=0$. Then $x(s)=0+\int_{s-1}^{s} \dot{x}<r$, a contradiction. 
(iii) Set $x:=x^{a^{+}}$. We have $A<x<-r / 2$ on $\left(-\infty, t\left(a^{+}\right)\right), \lim _{t \rightarrow-\infty} x(t)=A$ and $x\left(t\left(a^{+}\right)\right)=-r / 2$, and $0<\dot{x}$ on $\left(-\infty, t\left(a^{+}\right)\right)$. It follows that there exist $t_{1}<t_{2}<t\left(a^{+}\right)$ with $x\left(t_{1}\right)=\xi_{1}, x\left(t_{2}\right)=\xi_{2} \cdot\left|x_{0}-\phi_{A}\right|<r$ and $A+2 r<\xi_{1}$ give $0<t_{1}$. (H6) yields

$$
x\left(t_{1}+1\right)=\xi_{1}+\int_{t_{1}}^{t_{1}+1} \bar{f}(x(s-1)) d s \leqslant \xi_{2},
$$

therefore $t_{1}+1 \leqslant t_{2}$ and $\xi_{1} \leqslant x \leqslant \xi_{2}$ on $\left[t_{2}-1, t_{2}\right]$. Furthermore,

$$
x\left(t_{2}+1\right)=\xi_{2}+\int_{t_{2}}^{t_{2}+1} \bar{f}(x(s-1)) d s>B+r
$$

(see (H6)). (H3) shows $-r / 2<\dot{x}$, and we obtain $B<B+(r / 2)<x$ on the interval $\left[t_{2}+1, t_{2}+2\right]$.

Corollary 1. $a^{+} \in A^{+}, a^{-} \in A^{-}$.

Proposition 7. $A^{-} \cap A^{+}=\varnothing$.

Proof. (a) Let $a \in A^{+}$. Then $x_{t}^{a}>B$ for some $t>0$. Assume there exists $s>t$ with $x^{a}(s)=B, x^{a}>B$ on $[t-1, s)$. Then $\dot{x}^{a}(s) \leqslant 0 .-r / 2<\dot{x}^{a}$ gives $(B<)$ $x^{a}<B+r / 2$ in $[s-1, s)$. Hence $\dot{x}^{a}(s)>0$, a contradiction. It follows that $x^{a}>B$ on $[t-1, \infty)$.

(b) Let $a \in A^{-}$. Then $-r / 2<x_{t}^{a}<B-r / 2$ for some $t>0$. Assume there exists $s>t$ with $x^{a}(s) \in\{-r / 2, B\}$, and $-r / 2<x^{a}<B$ on $[t-1, s)$.

Case I: $x^{a}(s)=-r / 2$. Then $\dot{x}^{a}(s) \leqslant 0 .-r / 2<\dot{x}^{a}$ implies $x^{a}<0$ on $[s-1, s]$; $-r / 2<x^{a}(s-1)<0 ; 0<\dot{x}^{a}(s)$, a contradiction.

Case II: $x^{a}(s)=B$. Then $\dot{x}^{a}(s) \geqslant 0$. There exists $s^{\prime} \in(t, s)$ such that $x^{a}\left(s^{\prime}\right)=B$ $-r / 2$ and $B-r / 2<x^{a}<B$ in $\left(s^{\prime}, s\right)$. If $s^{\prime}+1 \leqslant s$, then $\dot{x}^{a}(s)=a f\left(x^{a}(s-1)\right)$ $<0$, a contradiction. If $s \in\left(s^{\prime}, s^{\prime}+1\right)$, then $\sigma-1 \in[t-1, s)$ for all $\sigma \in\left[s^{\prime}, s\right]$ which gives $-r / 2<x^{a}(\sigma-1)<B$, and $\dot{x}^{a}(\sigma)<r / 2$. This implies $r / 2=x^{a}(s)-$ $x^{a}\left(s^{\prime}\right)=\int_{s^{\prime}}^{s} \dot{x}^{a}<r\left(s-s^{\prime}\right) / 2<r / 2$, a contradiction.

It follows that $-r / 2<x^{a}<B$ on $[t, \infty)$.

(c) (a) and (b) imply $A^{-} \cap A^{+}=\varnothing$.

Corollary 2. The set $H:=A_{1}-\left(A^{-} \cup A^{+}\right)$is not empty and compact.

For the proof of Proposition 14 in $\$ 4$ we also need

REMARK 2. For every solution $x: \mathbf{R} \rightarrow \mathbf{R}$ of equation $(a), a \in A_{1}$, with $x_{t}>B$ for some $t \in \mathbf{R}, x(s)$ does not converge to $B$ as $s$ tends to $+\infty$.

Proof. We may proceed as in the proof of Proposition 7 and find $x>B$ on $[t-1, \infty)$. Suppose $\lim _{s \rightarrow+\infty} x(s)=B$. Then there exists $s \in \mathbf{R}$ with $B<x<B+r$ on $[s, \infty)$. But now, $\dot{x}>0$ on $[s+1, \infty)$ which leads to a contradiction.

Proposition 8. For every $a \in H$ there exists $z(a) \in \mathbf{R}$ such that $x^{a}<0$ in $(-\infty, z(a))$ and $x^{a}(z(a))=0$. We have $0<\dot{x}^{a}$ in $[z(a), z(a)+1)$ and $B-r<x^{a}$ $<B+\operatorname{rin}[z(a)+1, \infty)$.

Proof. (a) $a \in H$ implies $x^{a}(t(a)+1) \geqslant B-r / 2$ : In case $x^{a}(t(a)+1)<B-$ $r / 2,-r / 2<x^{a}<B-r / 2$ on $(t(a), t(a)+1]$, and there exists $s>t(a)+1$ with $-r / 2<x_{s}^{a}<B-r / 2$, or $a \in A^{-}$. 
(b) We have $\dot{x}^{a}>0$ in $[t(a), t(a)+1]$ so that there exists $z(a) \in(t(a), t(a)+1)$ with $x^{a}(z(a))=0$ and $x^{a}<0$ on $[t(a), z(a))$. It follows that $A<x^{a}<0$ on $(-\infty, z(a))$. Hence $\dot{x}^{a}>0$ on $[z(a), z(a)+1)$, and $x^{a}(z(a)+1) \geqslant x^{a}(t(a)+1) \geqslant$ $B-r / 2$.

(c) Assume $x^{a}(t) \geqslant B+r$ for some $t \geqslant z(a)+1$. Then $x^{a}>B+r / 2$ on $[t, t+1]$, or $a \in A^{+}$, a contradiction.

(d) Assume $x^{a}(t) \leqslant B-r$ for some $t \geqslant z(a)+1$. There exists $s>z(a)+1$ with $x^{a}(s)=B-r$ and $x^{a}>B-r$ on $[z(a)+1, s)$. We have $x^{a}>0$ on $(z(a), z(a)+1]$. Therefore $x^{a}>0$ on $(z(a), s) .-r / 2<\dot{x}^{a}$ implies $x^{a}<B-r / 2$ on $[s-1, s]$. It follows that $a \in A^{-}$, a contradiction.

Proposition 9. $\lim _{t \rightarrow+\infty} x^{a}(t)=B$ for every $a \in H$.

Proof. Let $a \in H$. Set $x:=x^{a}, z:=z(a)$. We have $\dot{x}>0$ in $[z, z+1)$.

(a) $x(z+1)>B$. Proof: $x(z+1) \leqslant B$ and equation $(a)$ imply that either $0<x$ $<B$ on $(z+1, \infty)$, or that there is a zero $z^{\prime}>z+1$ of $x$ with $-r / 2<\dot{x}<0$ on $\left(z+1, z^{\prime}+1\right)$. In the first case, $x(t)$ decreases to 0 as $t$ goes to $+\infty$. In both cases one obtains $a \in A^{-}$, a contradiction.

(b) It follows that there exists $b_{1} \in(z, z+1)$ with $x<B$ on $\left(z, b_{1}\right), x\left(b_{1}\right)=B$, $B<x<B+r$ on $\left(b_{1}, z+1\right)$. On $\left(z+1, b_{1}+1\right), \dot{x}<0$.

(c) $x\left(b_{1}+1\right)<B$. Proof: Assume $B \leqslant x\left(b_{1}+1\right)$. Then $B<x<B+r$ on $\left(b_{1}\right.$, $\left.b_{1}+1\right)$, and consequently $0<\dot{x}$ in $\left(b_{1}+1, b_{1}+2\right)$. We find some $t>b_{1}+1, t$ close to $b_{1}+1$, with $B<x$ on $[t, t+1]$. But this means $a \in A^{+}$, a contradiction.

(d) It follows that there exists $b_{2} \in\left(z+1, b_{1}+1\right)$ with $B<x<B+r$ in $\left[z+1, b_{2}\right), x\left(b_{2}\right)=B, B-r<x<B$ in $\left(b_{2}, b_{1}+1\right]$. Obviously, $0<\dot{x}$ in $\left(b_{1}+\right.$ $\left.1, b_{2}+1\right)$.

(e) $B<x\left(b_{2}+1\right)<B+r$. Proof: Compare the argument in (a).

(f) Proceeding by induction one finds a sequence $\left(b_{n}\right)$ with the following properties:

$$
\begin{aligned}
& b_{1}<z+1<b_{2}<b_{1}+1, \\
& B<x \leqslant x(z+1)<B+r \text { in }\left(b_{1}, b_{2}\right) \text {, } \\
& b_{n}<b_{n+1}<b_{n}+1<b_{n+2} \text { for all } n \in \mathbf{N} \text {, } \\
& \left.\begin{array}{l}
B-r<x\left(b_{n}+1\right) \leqslant x<B \\
B<x \leqslant x\left(b_{n}+1\right)<B+r
\end{array}\right\} \quad \text { in }\left(b_{n+1}, b_{n+2}\right) \text { for } n \in \mathbf{N}\left\{\begin{array}{l}
\text { odd } \\
\text { even. }
\end{array}\right.
\end{aligned}
$$

(g) The local extrema $x\left(b_{n+1}+1\right), n \geqslant 2$, satisfy

$$
\begin{aligned}
\left|x\left(b_{n+1}+1\right)-B\right| & =\left|x\left(b_{n+1}+1\right)-x\left(b_{n+2}\right)\right| \\
& \leqslant \int_{b_{n+2}-1}^{b_{n+1}} a|f \circ x| \leqslant \int_{b_{n}}^{b_{n+1}} a|f \circ x| \\
& \leqslant \int_{b_{n}}^{b_{n+1}} q|x(s)-B| d s \quad\left(\text { with } b_{n}<b_{n+2}-1\right) \\
& \leqslant q\left|x\left(b_{n-1}+1\right)-B\right| .
\end{aligned}
$$




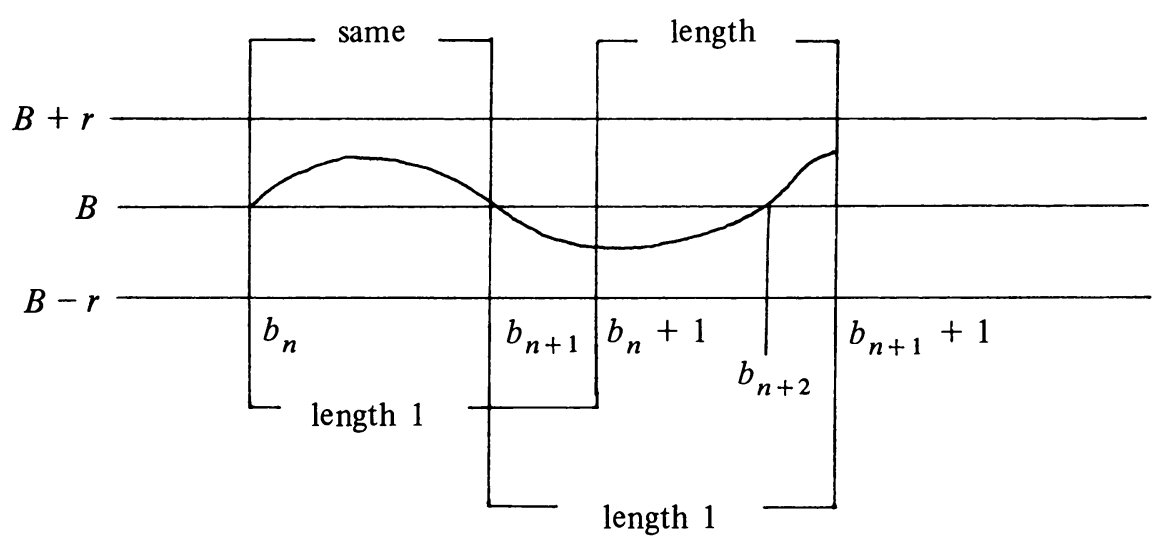

FIGURE 3

Here we have used (H1), (H4) and $|x-B|<r$ in $\left[b_{1}, \infty\right), a \leqslant a^{+}$. The inequalities obtained above imply $\lim _{t \rightarrow+\infty} x(t)=B$.

We set $a_{0}:=\max H<a^{+}$. Then $a \in A^{+}$for every $a \in\left(a_{0}, a^{+}\right]$. We shall prove that for $a>a_{0}$ sufficiently close to $a_{0}$ there exists a periodic solution of the second kind which is close to the union of the orbits of the heteroclinic solutions $x^{a_{0}}+$ $j(B-A), j \in \mathbf{Z}$.

3. Convergence to the unstable manifold. It is convenient to represent the sets $U_{a}(A), S_{a}(A)$ for $a$ close to $a_{0}$ as graphs of maps which are defined on fixed domains in the spaces $P_{0}:=P_{a_{0}}$ and $Q_{0}:=Q_{a_{0}}$. For this, and for several estimates which we need, we adapt results of Chafee [2] to the equation

$$
\dot{w}(t)=a h(w(t-1))
$$

with the shifted nonlinearity $h, h(\xi)=f(\xi+A)=f(\xi+B)$ for $\xi \in \mathbf{R}$, so that $h(0)=0$.

We write $u_{0}, \Phi_{0}, \Psi_{0}, p_{0}, q_{0},(\cdot, \cdot)_{0}$ instead of $u\left(a_{0}\right), \ldots,(\cdot, \cdot)_{a_{0}}$. Set $\chi(\theta):=0$ for $-1 \leqslant \theta<0$ and $\chi(0):=1, Y_{0}:=\left(\Psi_{0}, \chi\right)_{0}=\Psi_{0}(0)$.

THEOREM 1. There exist positive constants $\mu, u_{1}, u_{2}, u_{3}$ and $k_{2} \geqslant k_{1}$, a continuous function $h_{\mu}: \mathbf{R} \rightarrow \mathbf{R}$ with $h_{\mu}=h$ on $[-\mu, \mu]$, an open interval $A_{2}$ with $a_{0} \in A_{2} \subset A_{1}$, and continuous maps $u: \mathbf{R} \times A_{2} \rightarrow Q_{0}, s: Q_{0} \times A_{2} \rightarrow \mathbf{R}$ such that for every $a \in A_{2}$ we have:

(i) $u(0, a)=0$ and $\left|u(y, a)-u\left(y^{\prime}, a\right)\right| \leqslant\left|y-y^{\prime}\right|$ for all $y, y^{\prime} \in \mathbf{R}, s(0, a)=0$ and $\left|s(\phi, a)-s\left(\phi^{\prime}, a\right)\right| \leqslant\left|\phi-\phi^{\prime}\right|$ for $\phi, \phi^{\prime} \in Q_{0}$.

(ii) For every $\phi \in \bar{U}_{a}:=\left\{y \Phi_{0}+u(y, a) \mid y \in \mathbf{R}\right\}$ there exists a solution $w^{*}=$ $w^{*}(\phi, a): \mathbf{R} \rightarrow \mathbf{R}$ of equation

$$
\left(a h_{\mu}\right) \quad \dot{w}(t)=a h_{\mu}(w(t-1))
$$

with $w_{0}=\phi$ and $w_{t} \in \bar{U}_{a}$ for every $t \in \mathbf{R}$. Moreover, $w_{t}^{*}=y(t) \Phi_{0}+u(y(t), a)$ for every $t \in \mathbf{R}$ with the solution $y: \mathbf{R} \rightarrow \mathbf{R}$ of the initial value problem

$$
\dot{y}=u_{0} y+Y_{0} X\left(y \Phi_{0}+u(y, a), a\right), \quad y(0)=\left(\Psi_{0}, \phi\right)_{0},
$$

where $X(\bar{\phi}, a)=a \bar{\phi}(-1)-a_{0} \bar{\phi}(-1)+a h_{\mu}(\bar{\phi}(-1))-a \bar{\phi}(-1)$ for every $\bar{\phi} \in C$. 
(iii) $u_{0}<u_{1}<u_{2}<u_{3}<-\log a$, and

$$
|z(t, \phi, a)-u(y(t, \phi, a), a)| \leqslant k_{2} e^{-u_{2} t}|\phi|
$$

for all $t \geqslant 0$ and all $\phi \in C$, where $z(t, \phi, a):=q_{0} w_{t}, y(t, \phi, a):=\left(\Psi_{0}, w_{t}\right)_{0}$ with the solution $w:[-1, \infty) \rightarrow \mathbf{R}$ of $\left(a h_{\mu}\right)$ with $w_{0}=\phi$.

(iv) $\left|w_{t}\right| \leqslant k_{2} e^{-u_{3} t}\left|w_{0}\right|$ for every $t \geqslant 0$ and every solution $w:[-1, \infty) \rightarrow \mathbf{R}$ of $\left(a h_{\mu}\right)$ with $w_{0} \in \bar{S}_{a}:=\left\{s(\phi, a) \Phi_{0}+\phi \mid \phi \in Q_{0}\right\}$.

We write $\bar{U}_{0}:=\bar{U}_{a_{0}}, \bar{S}_{0}:=\bar{S}_{a_{0}}$. Statement (i) for $u$ and statement (ii) of Theorem 1 are proved in the same way as Theorem 4.1 of [2]. The proof of (iii) parallels the proof of Lemma 4.2.1 and of the resulting Theorem 4.2 of [2]. Part (i) as regards $s$, and part (iv) correspond to [2, Theorem 6.1].

Note that (iii) tells the rate of convergence of solutions to the "graph" of $u(\cdot, a)$ : The left-hand side of the estimate equals

$$
\left|w_{t}-\left(y(t, \phi, a) \Phi_{0}+u(y(t, \phi, a), a)\right)\right| .
$$

Corollary 3. There are constants $k_{3} \geqslant k_{2}$ and $\delta_{4} \in\left(0, \delta_{3}\right)$ and an open interval $A_{3} \subset A_{2}, a_{0} \in A_{3}$, such that for all $a \in A_{3}$, all $\phi \in \bar{U}_{a} \cap B\left(\delta_{4}\right)$ and all $t \leqslant 0$, $\left|w_{t}^{*}(\phi, a)\right| \leqslant k_{3} e^{u_{0} t / 2}|\phi|$.

Proof. Choose $\delta_{4}^{\prime}>0$ and an open interval $A_{3} \ni a_{0}$ in $A_{2}$ so small that

$$
\left|Y_{0} X\left(y \Phi_{0}+u(y, a), a\right)\right| \leqslant\left(u_{0} / 2\right)|y|
$$

for all $a \in A_{3}$ and all $y \in\left(-\delta_{4}^{\prime}, \delta_{4}^{\prime}\right)$. Let $y_{0} \in\left(-\delta_{4}^{\prime}, \delta_{4}^{\prime}\right), a \in A_{3}$. Consider the maximal solution $y$ of the initial value problem

$$
\dot{y}=u_{0} y+Y_{0} X\left(y \Phi_{0}+u(y, a), a\right), \quad y(0)=y_{0} .
$$

For $t \leqslant 0$ with $|y|<\delta_{4}^{\prime}$ in $[t, 0]$ and for $t \leqslant s \leqslant 0$, the variation-of-constants formula yields

$$
|y(s)| \leqslant e^{u_{0} s}\left|y_{0}\right|+\int_{s}^{0} e^{u_{0}(s-\sigma)}\left(u_{0} / 2\right)|y(\sigma)| d \sigma,
$$

and Gronwall's lemma implies $|y(s)| \leqslant e^{u_{0} s / 2}\left|y_{0}\right| \leqslant\left|y_{0}\right|$. It is now easy to obtain $|y(t)| \leqslant e^{u_{0} t / 2}\left|y_{0}\right|$ for all $t \leqslant 0$. Choose $\delta_{4}>0$ with $\left|\left(\Psi_{0}, \phi\right)_{0}\right|<\delta_{4}^{\prime}$ for $|\phi| \leqslant \delta_{4}$, $\delta_{4}<\delta_{3}$. For $\phi \in \bar{U}_{a} \cap B\left(\delta_{4}\right)$ and $y_{0}=\left(\Psi_{0}, \phi\right)_{0}$, we find

$$
\begin{aligned}
|y(t)| & \leqslant e^{u_{0} t / 2}|y(0)| \leqslant e^{u_{0} t / 2}\left|\left(\Psi_{0}, \phi\right)_{0}\right| \\
& \leqslant e^{u_{0} t / 2}\left(1+a_{0}\right) \Psi_{0}(0)|\phi|
\end{aligned}
$$

for every $t \leqslant 0$. Finally, we may use $\left|w_{t}^{*}(\phi, a)\right| \leqslant 2|y(t)|$ for all $t \in \mathbf{R}$.

In order to avoid a reference to a result with a lengthy proof in [2] we show

Corollary 4. There exist $\delta_{5} \in\left(0, \min \left\{\mu, \delta_{4}\right\}\right)$ and an open interval $A_{4} \subset A_{3}$ with $a_{0} \in A_{4}$ such that for every $a \in A_{4}$ and for every solution $w:[-1, \infty) \rightarrow \mathbf{R}$ of $($ ah $)$ with $|w|<\delta_{5}$, we have $w_{0} \in \bar{S}_{a}$. 
Proof. (a) The definition of $h$ and the properties of equation (a) imply that for every $a \in A_{1}$ and for every solution $w:[-1, \infty) \rightarrow \mathbf{R}$ of $(a h)$ with $|w| \leqslant \delta_{2}, w_{0}=\phi+$ $s_{a}(\phi)$ for some $\phi \in Q_{a} \cap B\left(\delta_{1}\right)$.

(b) Set $\delta_{5}^{\prime}:=\left(1 / k_{2}\right) \min \left\{\mu, \delta_{2}\right\}$. Let $a \in A_{2}$. We claim $\left\{\phi+s(\phi, a) \Phi_{0} \mid \phi \in Q_{0} \cap\right.$ $\left.B\left(\delta_{5}^{\prime}\right)\right\} \subset\left\{\psi+s_{a}(\psi) \mid \psi \in Q_{a} \cap B\left(\delta_{1}\right)\right\}$. Proof: The solution $\bar{w}:[-1, \infty) \rightarrow \mathbf{R}$ of $\left(a h_{\mu}\right)$ with $\bar{w}_{0}=\phi+s(\phi, a) \Phi_{0}, a \in A_{2}, \phi \in Q_{0} \cap B\left(\delta_{5}^{\prime}\right)$ satisfies $\left|\bar{w}_{t}\right| \leqslant \min \left\{\mu, \delta_{2}\right\}$ on $[0, \infty)$ (see Theorem 1(iv)). It follows that it is also a solution to $(a h)$, with $\bar{w}_{0}=\psi+s_{a}(\psi)$ for some $\psi \in Q_{a} \cap B\left(\delta_{1}\right)$ (see (a)).

(c) Choose $\delta_{5} \in\left(0, \min \left\{\mu, \delta_{4}\right\}\right)$ with $\left|q_{0}\right| \delta_{5}<\delta_{5}^{\prime}$, and an open interval $A_{4} \subset A_{3}$ with $a_{0} \in A_{4}$ and $\left|p_{a}-p_{0}\right| \leqslant 1 /\left(4\left(L_{1}+1\right)\right)$ for $a \in A_{4}$. Let $a \in A_{4}$. Consider $\phi \in B\left(\delta_{5}\right)$ with $\phi=\psi+s_{a}(\psi)$ for some $\psi$ in $Q_{a} \cap B\left(\delta_{1}\right)$. We show $\phi=\bar{\phi}+$ $s(\bar{\phi}, a) \Phi_{0}$ for $\bar{\phi}:=q_{0} \phi:$ First, $|\bar{\phi}| \leqslant\left|q_{0}\right||\phi|<\delta_{5}^{\prime}$. Hence $\bar{\phi}+s(\bar{\phi}, a) \Phi_{0}=\bar{\psi}+s_{a}(\bar{\psi})$ for some $\bar{\psi} \in Q_{a} \cap B\left(\delta_{1}\right)$ (see (b)). Suppose $\bar{\psi} \neq \psi$. With $q_{0}\left(\psi+s_{a}(\psi)\right)=q_{0}(\phi)=$ $\bar{\phi}=q_{0}\left(\bar{\phi}+s(\bar{\phi}, a) \Phi_{0}\right)=q_{0}\left(\bar{\psi}+s_{a} \bar{\psi}\right)$, it follows that $\psi-\bar{\psi}+s_{a}(\psi)-s_{a}(\bar{\psi})=$ $p_{0}\left(\psi+s_{a}(\psi)\right)-p_{0}\left(\bar{\psi}+s_{a}(\bar{\psi})\right)$. Hence

$$
\begin{aligned}
\psi-\bar{\psi} & =p_{a}\left(\bar{\psi}+s_{a}(\bar{\psi})\right)-p_{a}\left(\psi+s_{a}(\psi)\right)+p_{0}\left(\psi+s_{a}(\psi)\right)-p_{0}\left(\bar{\psi}+s_{a}(\bar{\psi})\right) \\
& =\left(p_{a}-p_{0}\right)(\bar{\psi}-\psi)+\left(p_{a}-p_{0}\right)\left(s_{a}(\bar{\psi})-s_{a}(\psi)\right),
\end{aligned}
$$

$0 \neq|\psi-\bar{\psi}| \leqslant \frac{1}{4}|\psi-\bar{\psi}|+\frac{1}{4}|\psi-\bar{\psi}|$, a contradiction. Therefore $\psi=\bar{\psi}$, or $\phi=\psi+$ $s_{a}(\psi)=\bar{\psi}+s_{a}(\bar{\psi})=\bar{\phi}+s(\bar{\phi}, a) \Phi_{0}$ with $\bar{\phi} \in Q_{0} \cap B\left(\delta_{5}^{\prime}\right)$.

(d) Let $a \in A_{4}$, and consider a solution $w:[-1, \infty) \rightarrow \mathbf{R}$ of $(a h)$ with $|w|<\delta_{5}$. Then $|w|<\delta_{2}$, and consequently, $w_{0}=\psi+s_{a}(\psi)$ for some $\psi \in Q_{a} \cap B\left(\delta_{1}\right)$. Now use (c) to complete the proof.

The following series of propositions prepares the application of the inequality $\operatorname{Re} \lambda<-u(a)$ for eigenvalues $\lambda \neq u(a)$ of $G_{a}, a \in A_{1}$.

Proposition 10. There exist constants $k_{4} \geqslant k_{3}, \delta_{6} \in\left(0, \delta_{5}\right)$ and an open interval $A_{5} \subset A_{4}$ with $a_{0} \in A_{5}$ such that for every $a \in A_{5}$, for every $T \geqslant 0$ and for every pair of solutions $w, \bar{w}:[-1, \infty) \rightarrow \mathbf{R}$ of $\left(\right.$ ah) with $|w| \leqslant \delta_{6}$ and $|\bar{w}| \leqslant \delta_{6}$ on $[-1, T]$, we have $\left|w_{t}-\bar{w}_{t}\right| \leqslant k_{4} e^{u_{1} t}\left|w_{0}-\bar{w}_{0}\right|$ for all $t \in[0, T]$.

Proof. This is an application of Gronwall's lemma to the variation-of-constants formula for ( $a h)$, for $a$ close to $a_{0}$. Note that the spectral radius of $G_{a_{0}}$ is given by $u_{0}$ so that

$$
\left|T_{a_{0}}(t, \phi)\right| \leqslant k_{4} e^{\left(u_{0}+\left(u_{1}-u_{0}\right) / 2\right) t}|\phi|
$$

for every $t \geqslant 0, \phi \in C$, with some constant $k_{4} \geqslant 1$. It is then convenient to write $a h(\xi)=a_{0} \xi+\left(a \xi-a_{0} \xi+a(h(\xi)-\xi)\right)$ for $a, \xi \in \mathbf{R}$, and to consider $\xi \rightarrow(\cdots)$ as a perturbation of $a_{0}$ id. The details are left to the reader.

Proposition 11. There are positive constants $\delta_{7}, \delta_{8}$ with $\delta_{8}<\delta_{7}$ and $2\left|p_{0}\right| k_{4} \delta_{8}<$ $\delta_{7}<\delta_{6} / 2 k_{3}$ such that for every $a \in A_{5}$ and for every solution $w:[-1, \infty) \rightarrow \mathbf{R}$ of $($ ah $)$ with $w_{0}=\phi \in B\left(\delta_{8}\right)-\bar{S}_{a}$ there exists $T=T(\phi, a) \geqslant 0$ with $\left|p_{0} w_{T}\right|=\delta_{7},\left|p_{0} w_{t}\right|<\delta_{7}$ on $[0, T),|w| \leqslant \delta_{6}$ on $[-1, T]$. 
Proof. (a) Take $\delta_{7}$ and $\delta_{8}$ with $k_{4} \delta_{8}<\delta_{6}, 2\left|p_{0}\right| k_{4} \delta_{8}<\delta_{7}<\delta_{6} / 2 k_{4}, 2 \delta_{7}+k_{4} \delta_{8}$ $<\delta_{6}$.

(b) Let $a \in A_{5}, \phi \in B\left(\delta_{8}\right)-\bar{S}_{a}$. $\delta_{8}<\delta_{6}<\delta_{5}$, and Corollary 4 shows that there exists $T^{\prime}>0$ with $|w|<\delta_{6}$ on $\left[-1, T^{\prime}\right)$ and $\left|w_{T^{\prime}}\right|=\delta_{6}$ for the solution $w=w(\phi, a)$ : $[-1, \infty) \rightarrow \mathbf{R}$ of $(a h), w_{0}=\phi . \delta_{6}<\delta_{5}<\mu$ implies that, on $\left[-1, T^{\prime}\right], w$ coincides with a solution of $\left(a h_{\mu}\right)$, and Theorem 1(iii) gives $\left|q_{0} w_{T^{\prime}}-u\left(\left(\Psi_{0}, w_{T^{\prime}}\right)_{0}, a\right)\right| \leqslant k_{2} \delta_{8}$, hence $\left|q_{0} w_{T^{\prime}}\right| \leqslant k_{4} \delta_{8}+\left|p_{0} w_{T^{\prime}}\right|$. Here we have used $k_{2} \leqslant k_{4},|u(y, a)| \leqslant|y|$ for $(y, a) \in \mathbf{R} \times A_{2}, p_{0} w_{T^{\prime}}=\left(\Psi_{0}, w_{T^{\prime}}\right)_{0} \Phi_{0},\left|\Phi_{0}\right|=1$.

We claim $\delta_{7}<\left|p_{0} w_{T^{\prime}}\right|$. Proof: Suppose $\left|p_{0} w_{T^{\prime}}\right| \leqslant \delta_{7}$. Then $\delta_{6}=\left|w_{T^{\prime}}\right| \leqslant\left|p_{0} w_{T^{\prime}}\right|+$ $\left|q_{0} w_{T^{\prime}}\right| \leqslant \delta_{7}+k_{4} \delta_{8}+\delta_{7}<\delta_{6}$, a contradiction.

(c) We have $\left|p_{0} w_{0}\right|=\left|p_{0} \phi\right| \leqslant\left|p_{0}\right||\phi| \leqslant\left|p_{0}\right| \delta_{8} \leqslant 2\left|p_{0}\right| k_{4} \delta_{8}<\delta_{7}$. It follows that there exists $T=T(\phi, a) \in\left(0, T^{\prime}\right)$ such that $\left|p_{0} w_{T}\right|=\delta_{7}$ and $\left|p_{0} w_{t}\right|<\delta_{7}$ on $[0, T)$. Finally, $T<T^{\prime}$ gives $|w|<\delta_{6}$ on $[-1, T]$.

The estimate

$$
\begin{aligned}
|\phi| & \leqslant\left|q_{0} \phi\right|+\left|p_{0} \phi-s\left(q_{0} \phi, a\right) \Phi_{0}\right|+\left|s\left(q_{0} \phi, a\right) \Phi_{0}\right| \\
& \leqslant\left|q_{0} \phi\right|+\left|p_{0} \phi-s\left(q_{0} \phi, a\right) \Phi_{0}\right|+\left|q_{0} \phi\right| \text { for }(\phi, a) \in C \times A_{2}
\end{aligned}
$$

shows that we can choose $\delta_{9} \in\left(0, \delta_{8}\right)$ and $\eta_{1}>0$ so that $|\phi|<\delta_{8}$ for all $(\phi, a) \in C$ $\times A_{5}$ which satisfy $\left|q_{0} \phi\right| \leqslant \delta_{9}$ and $\left|p_{0} \phi-s\left(q_{0} \phi, a\right) \Phi_{0}\right| \leqslant \eta_{1}$.

Proposition 12. There exists a constant $k_{5} \geqslant k_{4}$ such that for every $(\eta, \phi, a) \in$ $\left(0, \eta_{1}\right) \times C \times A_{5}$ with $\left|q_{0} \phi\right| \leqslant \delta_{9}$ and $0<\left|p_{0} \phi-s\left(q_{0} \phi, a\right) \Phi_{0}\right| \leqslant \eta$, we have $\mid q_{0} w_{T}-$ $u\left(\left(\Psi_{0}, w_{T}\right)_{0}, a\right) \mid \leqslant k_{5} \eta^{u_{2} / u_{1}}$ for $T=T(\phi, a)$, with the solution $w=w(\phi, a):[-1, \infty)$ $\rightarrow \mathbf{R}$ of $($ ah $)$ and $w_{0}=\phi$.

Proof. (a) Let $(\eta, \phi, a)$ be given as above. Then $|\phi|<\delta_{8} .0<\left|p_{0} \phi-s\left(q_{0} \phi, a\right) \Phi_{0}\right|$ gives $\phi \notin \bar{S}_{a}$, and we may apply Proposition 11 to the solution $w=w(\phi, a)$ : $[-1, \infty) \rightarrow \mathbf{R}$ of $(a h)$ with $w_{0}=\phi$.

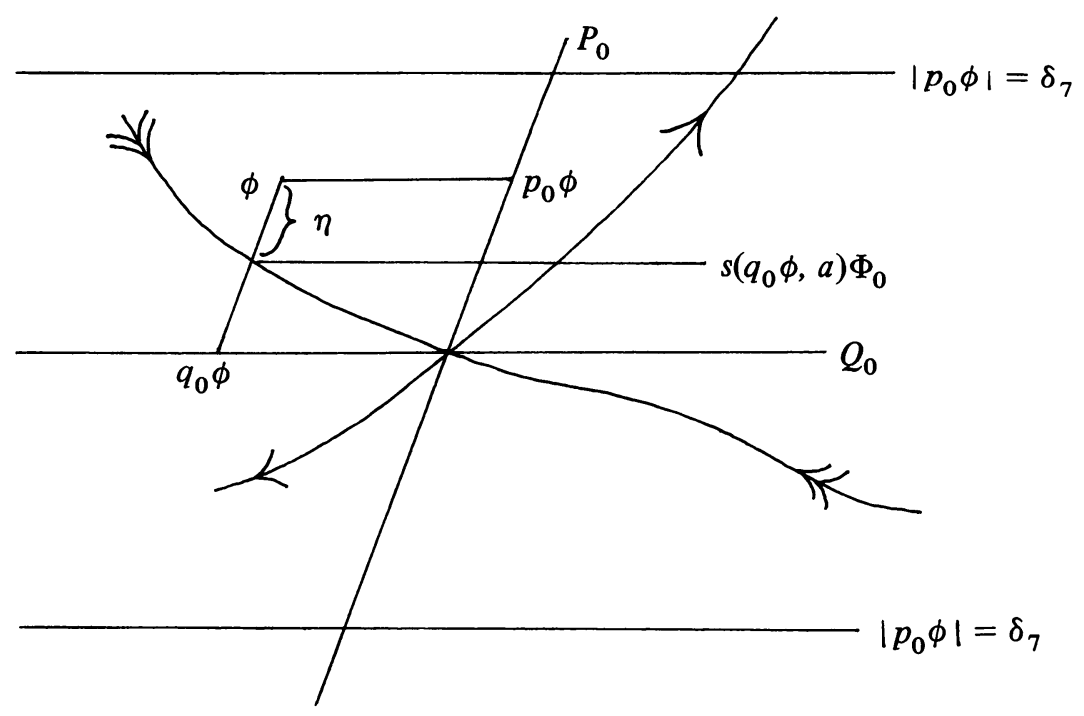

Figure 4 
(b) Set $\psi:=q_{0} \phi+s\left(q_{0} \phi, a\right) \Phi_{0} \in \bar{S}_{a}$. Then $\left|q_{0} \psi\right|=\left|q_{0} \phi\right| \leqslant \delta_{9}$ and $\mid p_{0} \psi-$ $s\left(q_{0} \psi, a\right) \Phi_{0} \mid=0<\eta \leqslant \eta_{1}$, hence $|\psi|<\delta_{8}$. The solution $\bar{w}:[-1, \infty) \rightarrow \mathbf{R}$ of $\left(a h_{\mu}\right)$ with $\bar{w}_{0}=\psi$ satisfies $\left|\bar{w}_{t}\right| \leqslant k_{2} e^{-u_{3} t}|\psi| \leqslant k_{2} \delta_{8}$ for every $t \geqslant 0$. Because of $k_{2} \delta_{8} \leqslant k_{4} \delta_{8}$ $<\delta_{6}<\mu, \bar{w}$ is also a solution of $(a h)$, and we may apply Proposition 10 to $w$ and $\bar{w}$, on the interval $[0, T]$. We find

$$
\begin{aligned}
\delta_{7} & =\left|p_{0} w_{T}\right| \leqslant\left|p_{0}\right| k_{4} e^{u_{1} T}|\phi-\psi|+\left|p_{0} \bar{w}_{T}\right| \\
& \leqslant\left|p_{0}\right|\left(k_{4} e^{u_{1} T}|\phi-\psi|+k_{2} e^{-u_{3} T}|\psi|\right) \\
& \leqslant\left|p_{0}\right| k_{4} e^{u_{1} T} \eta+\left|p_{0}\right| k_{2} \delta_{8} \leqslant\left|p_{0}\right| k_{4} e^{u_{1} T} \eta+\delta_{7} / 2
\end{aligned}
$$

or

$$
\frac{1}{u_{1}} \log \frac{\delta_{7}}{2 k_{4}\left|p_{0}\right| \eta} \leqslant T, \quad-u_{2} T \leqslant \frac{u_{2}}{u_{1}} \log \frac{2 k_{4}\left|p_{0}\right| \eta}{\delta_{7}} .
$$

On $[-1, T],|w|<\delta_{6}<\mu$, and $w$ is also a solution of $\left(a h_{\mu}\right)$ so that Theorem 1 yields

$$
\left|q_{0} w_{T}-u\left(\left(\Psi_{0}, w_{T}\right)_{0}, a\right)\right| \leqslant k_{2} e^{-u_{2} T}|\phi| \leqslant k_{2} \delta_{8}\left(2 k_{4}\left|p_{0}\right| / \delta_{7}\right)^{u_{2} / u_{1}} \eta^{u_{2} / u_{1}}
$$

We may also achieve $|w|>0$ and $|\dot{w}|>0$ on $[T-1, T]$ for $w_{0}$ sufficiently small and not in the local stable manifold. This will serve as a transversality condition for the construction of the Poincare map in the next section.

Proposition 13. There exists $\delta_{10} \in\left(0, \delta_{9}\right)$ with the following properties.

(i) $(\phi, a) \in B\left(\delta_{10}\right) \times A_{5}$ implies $\left|q_{0} \phi\right|<\delta_{9}$ and $\left|p_{0} \phi-s\left(q_{0} \phi, a\right) \Phi_{0}\right|<\eta_{1}$.

(ii) For $(\phi, a) \in B\left(\delta_{10}\right) \times A_{5}$ with $0<\left|p_{0} \phi-s\left(q_{0} \phi, a\right) \Phi_{0}\right|$, the solution $w=$ $w(\phi, a):[-1, \infty) \rightarrow \mathbf{R}$ of $($ ah $)$ with $w_{0}=\phi$ and the number $T=T(\phi, a)$ satisfy $T \geqslant 1$ and either $w>0$ and $\dot{w}>0$ on $[T-1, T]$, or $w<0$ and $\dot{w}<0$ on $[T-1, T]$.

(iii) Let $a \in A_{5}$. The map $\left\{\phi \in B\left(\delta_{10}\right) \mid p_{0} \phi \neq s\left(q_{0} \phi, a\right) \Phi_{0}\right\} \ni \phi \rightarrow T(\phi, a) \in \mathbf{R}$ is continuous.

Proof. Choose $\delta_{10}$ with $0<\left(\left|p_{0}\right|+\left|q_{0}\right|\right) \delta_{10}<\min \left\{\delta_{9}, \eta_{1}\right\},(1+q)^{2} \delta_{10}\left|p_{0}\right|<\delta_{7}$. This implies (i).

For a solution $w=w(\phi, a):[-1, \infty) \rightarrow \mathbf{R}$ of $(a h)$ with $a \in A_{5}, w_{0}=\phi \in B\left(\delta_{10}\right)$, $0<\left|p_{0} \phi-s\left(q_{0} \phi, a\right) \Phi_{0}\right|<\eta_{1}$, the hypotheses of Proposition 11 are satisfied since $\left|q_{0} \phi\right|<\delta_{9}$ and $0<\left|p_{0} \phi-s\left(q_{0} \phi, a\right) \Phi_{0}\right|<\eta_{1}$, or $|\phi|<\delta_{8}$ and $\phi \notin \bar{S}_{a}$. We obtain $\left|p_{0} w_{T}\right|=\delta_{7},\left|p_{0} w_{t}\right|<\delta_{7}$ on $[0, T)$ for $T=T(\phi, a)$, and $\left|w_{t}\right|<\delta_{6}<r$ on $[0, T]$ (see the proof of Proposition 11).

(a) We claim $T \geqslant 1$. Proof: For $t \in[0,1]$,

$$
\begin{aligned}
|w(t)| & =\left|w(0)+\int_{0}^{t} \dot{w}\right| \leqslant \delta_{10}+\int_{-1}^{i-1}|a h \circ \phi| \\
& =\delta_{10}+\int_{-1}^{t-1}|a f(\phi(s)+A)| d s \leqslant \delta_{10}+q \delta_{10}<\delta_{7} /\left|p_{0}\right| \leqslant\left|w_{T}\right| .
\end{aligned}
$$

Together with $|\phi| \leqslant \delta_{10}<\delta_{7} /\left|p_{0}\right|$, we find $\sup _{[-1,1]}|w|<\left|w_{T}\right|$. Hence $T \geqslant 1$.

(b) $\left|w_{T-1}\right|>(1+q) \delta_{10}$ since $\left|w_{T-1}\right| \leqslant(1+q) \delta_{10}$ would imply

$$
\begin{aligned}
|w(t)| & =\left|w(T-1)+\int_{T-2}^{t-1} a h \circ w\right| \leqslant(1+q) \delta_{10}+q(1+q) \delta_{10} \\
& <\delta_{7} /\left|p_{0}\right| \leqslant\left|w_{T}\right|
\end{aligned}
$$

for every $t \in[T-1, T]$, a contradiction. 
(c) Take $s_{1} \in[-1, T-1]$ with $\left|w\left(s_{1}\right)\right|=\sup _{[-1, T-1]}|w|$. Because of (b) and $|\phi| \leqslant \delta_{10}$ there exists $s_{2} \in(0, T-1]$ with $\left|w\left(s_{2}\right)\right|=\sup _{[-1, T-1]}|w|$ and $|w|<\left|w\left(s_{2}\right)\right|$ on $\left[-1, s_{2}\right)$. We have $1 \leqslant s_{2}$ since $0<s_{2}<1$ would imply

$$
\begin{aligned}
0 & <\left|w\left(s_{2}\right)\right|=\left|w(0)+\int_{-1}^{s_{2}-1} a h \circ \phi\right| \\
& \leqslant \delta_{10}+q \delta_{10}<\left|w_{T-1}\right| \leqslant\left|w\left(s_{2}\right)\right|,
\end{aligned}
$$

a contradiction.

Claim: $w_{s_{2}}<0$ or $w_{s_{2}}>0$. Proof: Suppose $w_{s_{2}} \notin K \cup-K$. Then there is a zero $z \in\left[s_{2}-1, s_{2}\right]$ of $w, z \geqslant 0$. We find

$$
\begin{aligned}
0 & <\left|w\left(s_{2}\right)\right|=\left|\int_{z}^{s_{2}} \dot{w}\right|=\left|\int_{z-1}^{s_{2}-1} a h \circ w\right| \\
& =\left|\int_{z-1}^{s_{2}-1} a f(w(s)+A) d s\right| \leqslant q \sup _{\left[-1, s_{2}-1\right]}|w| \leqslant q\left|w\left(s_{2}\right)\right|,
\end{aligned}
$$

a contradiction.

(d) Altogether, $1 \leqslant s_{2}$ and $w_{s_{2}} \in K \cup-K$. Assume $w_{s_{2}} \in K$. Equation (ah) and the estimate $|w|<r$ on $[-1, T]$ imply $\dot{w}>0$ on $\left[s_{2}, T-1\right]$. This shows $w_{T-1} \in K$, and $\dot{w}>0$ on $[T-1, T]$, finally $w_{T} \in K$. For $w_{s_{2}}<0$, the analogous argument yields $w_{T}<0, \dot{w}<0$ on $[T-1, T]$.

(e) Proof OF (iii). Let $a \in A_{5}$ and $\psi \in B\left(\delta_{10}\right)$ be given with $p_{0} \psi \neq s\left(q_{0} \psi, a\right) \Phi_{0}$. Set $\bar{w}:=w(\psi, a)$. Assume $\bar{w}_{\bar{T}} \in K$ and $\dot{\bar{w}}>0$ on $[\bar{T}-1, \bar{T}]$ for $\bar{T}=T(\psi, a)$. Let $\varepsilon \in(0,1)$ be given so that $\bar{w}_{\bar{T}+\varepsilon}>\bar{w}_{\bar{T}}$. On $[0, \bar{T}-\varepsilon],\left|p_{0} \bar{w}_{t}\right|<\delta_{7}$, and $\left|p_{0} \bar{w}_{\bar{T}+\varepsilon}\right|=$ $\left(\Psi_{0}, \bar{w}_{\bar{T}+\varepsilon}\right)_{0}>\left(\Psi_{0}, \bar{w}_{\bar{T}}\right)_{0}=\delta_{7}$. Continuous dependence of solutions on initial values shows that there exists $\rho>0$ so that $\phi \in B\left(\delta_{10}\right)$ and $|\phi-\psi| \leqslant \rho$ imply $\left|p_{0} w_{t}\right|<\delta_{7}$ on $[0, \bar{T}-\varepsilon]$ and $\left|p_{0} w_{\bar{T}+\varepsilon}\right|>\delta_{7}$ for $w=w(\phi, a)$. This yields $T(\phi, a) \in[\bar{T}-\varepsilon, \bar{T}+\varepsilon]$ provided $p_{0} \phi \neq s\left(q_{0} \phi, a\right) \Phi_{0}$. The proof in case $\bar{w}_{\bar{T}} \in-K$ is similar.

4. Bifurcation of periodic solutions of the second kind. The initial values for the desired periodic solutions will arise as fixed points of Poincaré maps which are defined on subsets of the hyperplane $\phi_{A}+\delta_{7} \Phi_{0}+Q_{0}$. In order to construct these maps we rewrite results of $\S 2$ on the solutions $x^{a}$ in the unstable manifolds $U_{a}(A)$ now in terms of the reparametrized unstable manifolds of Theorem 1.

For $a \in A_{5}$, consider the function

$$
\tilde{x}^{a}: \mathbf{R} \ni t \rightarrow A+w^{*}\left(\delta_{7} \Phi_{0}+u\left(\delta_{7}, a\right), a\right)(t) \in \mathbf{R} .
$$

Corollary 3 shows $\left|w^{*}(\cdots)(t)\right| \leqslant k_{3} e^{u_{0} t / 2} 2 \delta_{7}$ on $(-\infty, 0]$. We have $2 k_{3} \delta_{7}<\delta_{6}<\mu$ (see Proposition 11). It follows that $\tilde{x}^{a}-A$ is a solution to equation (ah) for $t \leqslant 0$, and $\tilde{x}^{a}$ is a solution to equation $(a)$ on $(-\infty, 0]$. Moreover, $\tilde{x}_{0}^{a}=\phi_{A}+\delta_{7} \Phi_{0}+$ $u\left(\delta_{7}, a\right)$ and $\lim _{t \rightarrow-\infty} x^{a}(t)=A$ (Corollary 3). We define $\bar{x}^{a}: \mathbf{R} \rightarrow \mathbf{R}$ to be the solution of equation $(a)$ with $\bar{x}^{a}=\tilde{x}^{a}$ on $(-\infty, 0]$, and $\bar{x}^{0}:=\bar{x}^{a_{0}}$.

Proposition 14. (i) For every $a \in A_{5}$ and every $t \leqslant 0, \bar{x}_{t}^{a} \in \phi_{A}+K$.

(ii) There exists $t_{0} \geqslant 0$ with $\bar{x}_{t}^{0} \in \phi_{B}+\bar{S}_{0}$ for every $t \geqslant t_{0}$, and $\lim _{t \rightarrow+\infty} \bar{x}^{0}(t)=B$.

(iii) For every $a \in A_{5}$ with $a>a_{0}$ there exists $t>0$ with $\bar{x}_{t}^{a} \in \phi_{B}+K$, and $\bar{x}_{s}^{a} \notin \phi_{B}+\left\{\phi+s(\phi, a) \Phi_{0} \mid \phi \in Q_{0} \cap B\left(\mu / 2 k_{3}\right)\right\}$ for every $s \in \mathbf{R}$. 
Proof. (a) Proof of (i). Let $a \in A_{5}$. We have

$$
\left|\bar{x}^{a}(t)-A\right|=\left|w^{*}(\cdots)(t)\right| \leqslant 2 k_{3} \delta_{7} e^{u_{0} t / 2}<\delta_{6} e^{u_{0} t / 2}<r e^{u_{0} t / 2}
$$

on $(-\infty, 0]$, and Proposition 4 yields $\bar{x}_{0}^{a} \in \phi_{A}+(K \cup-K)$; note $\bar{x}_{0}^{a}-\phi_{A} \in \delta_{7} \Phi_{0}+$ $Q_{0}$, or $\bar{x}_{0}^{a} \neq \phi_{A} . \bar{x}_{0}^{a} \in \phi_{A}-K$ would imply $\delta_{7} \Phi_{0}=p_{0}\left(\bar{x}_{0}^{a}-\phi_{A}\right) \in-K$, a contradiction. Therefore $\bar{x}_{0}^{a} \in \phi_{A}+K$, and Proposition 4 gives $\bar{x}_{t}^{a} \in \phi_{A}+K$ for all $t \leqslant 0$.

(b) We show that for every $a \in A_{5}$ there exist $\sigma \leqslant 0$ and $s \leqslant 0$ with $x_{\sigma}^{a}=\bar{x}_{s}^{a}$ : Let $a \in A_{5} \cdot\left|\bar{x}_{t}^{a}-\phi_{A}\right| \leqslant \delta_{2}$ on $(-\infty, 0]$ implies $\bar{x}_{t}^{a}-\phi_{A}=\bar{\phi}_{t}+u_{a}\left(\bar{\phi}_{t}\right)$ for every $t \leqslant 0$, with some $\bar{\phi}_{t} \in P_{a}=\mathbf{R} \Phi_{a}$ (see the statement of the saddle point property in $\S 1$ and the choice of $\delta_{2}$ in §2). (i) and the monotonicity of $p_{a}$ (Proposition 3) give $\bar{\phi}_{t}=p_{a}\left(\bar{x}_{t}^{a}-\phi_{A}\right) \in K$ for $t \leqslant 0$. Moreover, $\lim _{t \rightarrow-\infty} \bar{\phi}_{t}=\lim _{t \rightarrow-\infty} p_{a}\left(\bar{x}_{t}^{a}-\phi_{A}\right)=0$.

Now consider the solutions $x^{a}$ from $§ 2$. Remark 1 says that $x_{t}^{a}-\phi_{A}=\phi_{t}+u_{a}\left(\phi_{t}\right)$ for every $t \leqslant 0$, with $\phi_{t} \in P_{a}$. Propositions 5 and 3 show $\phi_{t}=p_{a}\left(x_{t}^{a}-\phi_{A}\right) \in K$ for all $t \leqslant 0$. Also, $\phi_{t}=p_{a}\left(x_{t}^{a}-\phi_{A}\right)$ converges to 0 as $t$ tends to $-\infty$. Now $P_{a}=\mathbf{R} \Phi_{a}$ implies $p_{a}\left(x_{\sigma}^{a}-\phi_{A}\right)=\phi_{\sigma}=\bar{\phi}_{s}=p_{a}\left(\bar{x}_{s}^{a}-\phi_{A}\right)$ for some $\sigma \leqslant 0, s \leqslant 0$. Therefore, $x_{\sigma}^{a}=\bar{x}_{s}^{a}$, and $x^{a}(t+\sigma)=\bar{x}^{a}(t+s)$ for all $t \geqslant-1$.

(c) Proposition 9 and (b) show $\lim _{t \rightarrow+\infty} \bar{x}^{0}(t)=B$. There exists $t_{0} \geqslant 0$ with $\left|\bar{x}^{0}(s)-B\right|<\delta_{5}$ for $s \geqslant t_{0}-1$. Let $t \geqslant t_{0}$. We may apply Corollary 4 to the solution $-1 \leqslant s \rightarrow \bar{x}^{0}(t+s)-B$ of $\left(a_{0} h\right)$, and assertion (ii) is proved.

(d) Proof of (iii). Let $a \in A_{5}, a>a_{0}$. Then $a \in A^{+}$, and $x_{t}^{a}>B$ for some $t>0$. (b) shows $\bar{x}_{(t-\sigma)+s}^{a}=x_{t}^{a}>B$, with $\sigma \leqslant 0, s \leqslant 0$. The first argument in (a) above says that $\bar{x}^{a} \leqslant A+r<B$ on $(-\infty, 0]$. Therefore $(t-\sigma)+s$ must be positive, and the first part of assertion (iii) is shown. Remark 2 implies that $\bar{x}^{a}(t)$ does not converge to $B$ as $t$ tends to $+\infty$. Assume $\bar{x}_{s}^{a}=\phi_{B}+\phi+s(\phi, a) \Phi_{0}$ with $\phi \in Q_{0} \cap B\left(\mu / 2 k_{3}\right)$ for some $s \in \mathbf{R}$. The solution $w:[-1, \infty) \rightarrow \mathbf{R}$ of $\left(a h_{\mu}\right)$ with $w_{0}=\bar{x}_{s}^{a}-\phi_{B}=\phi+$ $s(\phi, a) \Phi_{0}$ satisfies $|w| \leqslant \mu$ and $\lim _{t \rightarrow+\infty} w(t)=0$ (see Theorem 1(iv)). It follows that $w$ is also a solution to $(a h)$. Hence $w(t)=\bar{x}^{a}(t+s)-B$ for all $t \geqslant 0$, and $\lim _{t \rightarrow+\infty} \bar{x}^{a}(t)=B$, a contradiction.

Now we are ready to construct Poincaré maps. Choose $\delta_{11}$ with $0<3 \delta_{11}<$ $\min \left\{\delta_{10}, \mu /\left(2 k_{3}\left|q_{0}\right|\right)\right\}$ and $\tau \geqslant 1$ with $\bar{x}_{\tau}^{0}-\phi_{B}$ in $\bar{S}_{0} \cap B\left(\delta_{11}\right)$. Then

$$
0=p_{0}\left(\bar{x}_{\tau}^{0}-\phi_{B}\right)-s\left(q_{0}\left(\bar{x}_{\tau}^{0}-\phi_{B}\right), a_{0}\right) \Phi_{0} .
$$

The continuity of the map $A_{5} \ni a \rightarrow \bar{x}_{\tau}^{a} \in C$ implies the existence of $a_{1}>a_{0}$ with $\left[a_{0}, a_{1}\right] \subset A_{5}$ and $\bar{x}_{\tau}^{a}-\phi_{B} \in B\left(2 \delta_{11}\right)$ for every $a$ in $\left[a_{0}, a_{1}\right]$. Proposition 14(iii) and the inequality $\left|q_{0}\left(\bar{x}_{\tau}^{a}-\phi_{B}\right)\right| \leqslant\left|q_{0}\right| 2 \delta_{11}<\mu / 2 k_{3}$ imply

$$
0 \neq p_{0}\left(\bar{x}_{\tau}^{a}-\phi_{B}\right)-s\left(q_{0}\left(\bar{x}_{\tau}^{a}-\phi_{B}\right), a\right) \Phi_{0} \text { for } a_{0}<a \leqslant a_{1} .
$$

We define $\eta(a):=\left|p_{0}\left(\bar{x}_{\tau}^{a}-\phi_{B}\right)-s\left(q_{0}\left(\bar{x}_{\tau}^{a}-\phi_{B}\right), a\right) \Phi_{0}\right|$ for $a_{0} \leqslant a \leqslant a_{1}$. The map $a \rightarrow \eta(a)$ is continuous with $\eta\left(a_{0}\right)=0<\eta(a)$ for $a_{0}<a \leqslant a_{1}$.

We know from Proposition 1, applied to equation $(a)$, that there exists a constant $k_{6} \geqslant k_{5}$ with $\left|x_{\tau}(\phi, a)-\bar{x}_{\tau}^{a}\right| \leqslant k_{6}\left|\phi-\bar{x}_{0}^{a}\right|$ for every $a$ in $\left[a_{0}, a_{1}\right]$ and for every $\phi \in C$. Of course, $x(\phi, a):[-1, \infty) \rightarrow \mathbf{R}$ denotes the solution of equation $(a)$ with $x_{0}(\phi, a)=\phi$.

We choose $\eta_{2} \in\left(0, \eta_{1}\right)$ so small that for $0<\eta \leqslant \eta_{2}, k_{6} k_{5}(3 \eta / 2)^{u_{2} / u_{1}}<\delta_{11}$ and $\left(\left|p_{0}\right|+\left|q_{0}\right|\right) k_{6} k_{5}(3 \eta / 2)^{u_{2} / u_{1}}<\eta / 2$. It is here that we exploit $u_{2}>u_{1}$ which was a 
consequence of the crucial hypothesis on the characteristic values of the linearized equation.

Next, we choose $a_{2} \in\left(a_{0}, a_{1}\right]$ so that for $a_{0}<a \leqslant a_{2}, \eta(a) \leqslant \eta_{2} / 2$. For this range of parameters $a$ we define

$$
D_{a}:=\left\{\phi \in C\left|p_{0}\left(\phi-\phi_{A}\right)=\delta_{7} \Phi_{0},\right| \phi-\bar{x}_{0}^{a} \mid \leqslant k_{5}(3 \eta(a) / 2)^{u_{2} / u_{1}}\right\}
$$

as the domain for a Poincaré map. Clearly $\bar{x}_{0}^{a} \in D_{a}$. $D_{a}$ is closed, bounded and convex.

Proposition 15. Let $a \in\left(a_{0}, a_{2}\right]$.

(i) We have $\left|x_{\tau}(\phi, a)-\phi_{B}\right|<\delta_{10}$ and

$$
0<\left|p_{0}\left(x_{\tau}(\phi, a)-\phi_{B}\right)-s\left(q_{0}\left(x_{\tau}(\phi, a)-\phi_{B}\right), a\right) \Phi_{0}\right| \leqslant 3 \eta(a) / 2
$$

for every $\phi \in D_{a}$.

(ii) The map $P_{a}: D_{a} \ni \phi \rightarrow x_{\tau+T(\psi, a)}(\phi, a)-\phi_{B}+\phi_{A} \in C$, where $\psi=\psi(\phi)=$ $x_{\tau}(\phi, a)-\phi_{B}$, is continuous.

(iii) $p_{0}\left(P_{a} \phi-\phi_{A}\right)=\delta_{7} \Phi_{0}$ for every $\phi \in D_{a}$.

Proof. (a) Proof of (i). $\phi \in D_{a}$ gives

$$
\left|x_{\tau}(\phi, a)-\bar{x}_{\tau}^{a}\right| \leqslant k_{6}\left|\phi-\bar{x}_{0}^{a}\right| \leqslant k_{6} k_{5}(3 \eta(a) / 2)^{u_{2} / u_{1}}<\delta_{11}
$$

hence

$$
\begin{aligned}
\left|x_{\tau}(\phi, a)-\phi_{B}\right| & \leqslant\left|x_{\tau}(\phi, a)-\bar{x}_{\tau}^{a}\right|+\left|\bar{x}_{\tau}^{a}-\phi_{B}\right| \\
& \leqslant \delta_{11}+2 \delta_{11}<\delta_{10} .
\end{aligned}
$$

Furthermore,

$$
\begin{aligned}
\mid\left[p_{0}\left(x_{\tau}(\phi, a)-\phi_{B}\right)-s\right. & \left.\left(q_{0}\left(x_{\tau}(\phi, a)-\phi_{B}\right), a\right) \Phi_{0}\right] \\
& -\left\{p_{0}\left(\bar{x}_{\tau}^{a}-\phi_{B}\right)-s\left(q_{0}\left(\bar{x}_{\tau}^{a}-\phi_{B}\right), a\right) \Phi_{0}\right\} \mid \\
\leqslant & \left|p_{0}\left(x_{\tau}(\phi, a)-\bar{x}_{\tau}^{a}\right)\right|+\left|q_{0}\left(x_{\tau}(\phi, a)-\bar{x}_{\tau}^{a}\right)\right| \\
\leqslant & \left(\left|p_{0}\right|+\left|q_{0}\right|\right) k_{6} k_{5}(3 \eta(a) / 2)^{u_{2} / u_{1}}<\eta(a) / 2 .
\end{aligned}
$$

The definition of $\eta(a)$ now implies $[\cdots] \neq 0$, and also $\| \cdots] \mid<3 \eta(a) / 2$.

(b) Proof of (ii). Assertion (i) and Proposition 13(ii) show that for $\phi \in D_{a}$ and $\psi$ as above, $T=T(\psi, a)$ is defined, with $\left|p_{0} w_{T}\right|=\delta_{7}$ and $\left|p_{0} w_{t}\right|<\delta_{7}$ on $[0, T)$ for the solution $w=w(\psi, a):[-1, \infty) \rightarrow \mathbf{R}$ of $(a h)$ with $w_{0}=\psi$. Moreover, $w_{T} \in K$ and $\dot{w}>0$ on $[T-1, T]$, or $w_{T} \in-K$ and $\dot{w}<0$ on $[T-1, T]$. We have $w(\psi, a)(t)=$ $x(\phi, a)(\tau+t)-B$ for every $t \geqslant-1$. It follows that the map $P_{a}$ above is well defined, with

$$
P_{a} \phi=x_{\tau+T(\psi, a)}-\phi_{B}+\phi_{A}=w_{T(\psi, a)}(\psi, a)+\phi_{A},
$$

$\psi=\psi(\phi)=x_{\tau}(\phi, a)-\phi_{B}$, for all $\phi \in D_{a}$. Proposition 13(iii), together with continuous dependence of solutions on initial values, implies continuity.

(c) We show $P_{a} \bar{x}_{0}^{a}-\phi_{A} \in K$. Proposition 14 gives $\bar{x}_{t}^{a}>\phi_{B}$ for some $t>0$. This yields $\bar{x}^{a}>B$ on $[t-1, \infty)$; compare the proof of Proposition 7 .

(ca) In case $0<t \leqslant \tau+T\left(\psi\left(\bar{x}_{0}^{a}\right), a\right), \bar{x}^{a}>B$ on $[\tau+T(\cdots)-1, \tau+T(\cdots)]$, or $P_{a} \bar{x}_{0}^{a}-\phi_{A}=\bar{x}_{\tau+T(\cdots)}^{a}-\phi_{B}>0$.

(cb) The case $\tau+T\left(\psi\left(\bar{x}_{0}^{a}\right), a\right)<t$. 
(cba) $B \leqslant \bar{x}^{a}(s)$ for some $s \in[\tau-1, \tau]$. Proof: Suppose $\bar{x}_{\tau}^{a}<B$. Then $B-r<$ $\bar{x}_{\tau}^{a}<B$ because of $\bar{x}_{\tau}^{a} \in \phi_{B}+B\left(2 \delta_{11}\right)$ and $2 \delta_{11}<\delta_{10}<r$.

(cbaa) $-r<\bar{x}^{a}$ on $[\tau-1, \infty)$. Proof: Suppose $\bar{x}^{a}(\hat{t}) \leqslant-r$ for some $\hat{t} \geqslant \tau-1$. $B-r<\bar{x}_{\tau}^{a}$ implies that there exists $\tilde{t}>\tau$ with $\bar{x}^{a}>-r$ on $[\tau-1, \tilde{t})$ and $\bar{x}^{a}(\tilde{t})=-r$. Hence $\dot{\bar{x}}^{a}(\tilde{t}) \leqslant 0$. On the other hand, $\dot{\bar{x}}^{a} \geqslant-r / 2$, hence $-r / 2 \geqslant \bar{x}^{a}(\tilde{t}-1)>-r$, and $\dot{\bar{x}}^{a}(\tilde{t})=a f\left(\bar{x}^{a}(\tilde{t}-1)\right)>0$, a contradiction.

(cbab) $\bar{x}^{a}<B$ on $[\tau-1, \infty)$. Proof: Suppose $\bar{x}^{a}(\hat{t})=B$ for some $\hat{t} \geqslant \tau-1$. $\bar{x}_{\tau}^{a}<B$ implies that there exists $\tilde{t}>\tau$ with $\bar{x}^{a}(\tilde{t})=B$ and $\bar{x}^{a}<B$ on $[\tau-1, \tilde{t})$. Then $\dot{\bar{x}}^{a}(\tilde{t}) \geqslant 0$. We have $\tilde{t} \geqslant \tau+1$ since $B-r<\bar{x}^{a}<B$ on $[\tau-1, \tau]$ gives $\dot{\bar{x}}^{a}<0$ on $[\tau, \tau+1] .-r<\bar{x}^{a} \leqslant B$ on $[\tilde{t}-2, \tilde{t}-1] \subset[\tau-1, \infty)$ shows $\dot{\bar{x}}^{a} \leqslant r / 2$ on $[\tilde{t}-1, \tilde{t}]$. Therefore $B>\bar{x}^{a}(\tilde{t}-1) \geqslant B-r / 2$, or $\dot{\bar{x}}^{a}(\tilde{t})=a f\left(\bar{x}^{a}(\tilde{t}-1)\right)<0$, a contradiction.

(cbac) The preceding property contradicts $\bar{x}^{a}>B$ on $[t-1, \infty)$, and assertion (cba) is proved.

(cbb) We have $P_{a} \bar{x}_{0}^{a}-\phi_{A} \in K \cup-K$ (see (b)). It remains to exclude

$$
\bar{x}_{\tau+T(\bar{\psi}, a)}^{a}<B, \quad \text { where } \bar{\psi}=\bar{\psi}\left(\bar{x}_{0}^{a}\right)
$$

in the case under investigation. Inequality (*) implies that there is some $s_{0} \in(s, \tau+$ $T(\bar{\psi}, a))$ with $\bar{x}^{a}\left(s_{0}\right)=B$ and $\bar{x}^{a}<B$ in $\left(s_{0}, \tau+T(\bar{\psi}, a)\right]$, and $s_{0}+1<\tau+$ $T(\bar{\psi}, a) . \dot{\bar{x}}^{a} \geqslant-r / 2$ gives $B>\bar{x}^{a} \geqslant B-r / 2$ on $\left(s_{0}, s_{0}+1\right]$. As in (cba), we infer $\bar{x}^{a}<B$ on $\left(s_{0}, \infty\right)$. This contradicts $\bar{x}^{a}>B$ on $[t-1, \infty)$.

(d) Proof of (iii). $P_{a}$ is continuous with $P_{a}\left(D_{a}\right) \subset\left(\phi_{A}+K\right) \cup\left(\phi_{A}-K\right)$ (see (b)). $D_{a}$ is connected. $\phi_{A}+K$ and $\phi_{A}-K$ are disjoint open subsets of $C$. (c) shows $P_{a}^{-1}\left(\phi_{A}+K\right) \neq \varnothing$. Then $P_{a}^{-1}\left(\phi_{A}-K\right)=\varnothing$, and $P_{a}\left(D_{a}\right) \subset \phi_{A}+K$.

Let $\phi \in D_{a}$. The monotonicity of $p_{0}$ now implies $p_{0}\left(P_{a} \phi-\phi_{A}\right) \in K$. We have $\left|p_{0}\left(P_{a} \phi-\phi_{A}\right)\right|=\delta_{7}\left(\right.$ see (b)), and it follows that $p_{0}\left(P_{a} \phi-\phi_{A}\right)=\delta_{7} \Phi_{0}$.

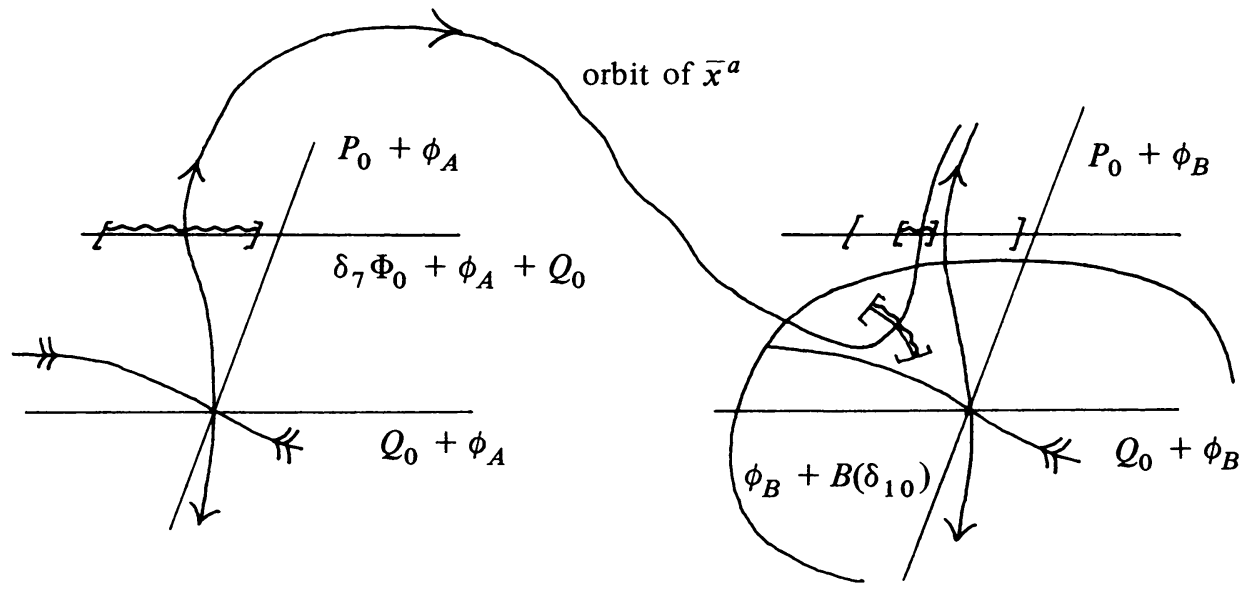

FIGURE 5 
Corollary 5. For every $a \in\left(a_{0}, a_{2}\right], P_{a}$ has a fixed point $\phi_{a}$, and there exists $a$ periodic solution of the second kind $x(a): \mathbf{R} \rightarrow \mathbf{R}$ of equation $(a)$ with $x(a) \|[-1,0]=$ $\phi_{a} \in D_{a}$.

Proof. (a) We show $P_{a}\left(D_{a}\right) \subset D_{a}$ : Let $\phi \in D_{a}$. It remains to prove that $\mid P_{a} \phi-$ $\bar{x}_{0}^{a} \mid \leqslant k_{5}(3 \eta(a) / 2)^{u_{2} / u_{1}}$. Proposition 15(i) says that $\psi:=x_{\tau}(\phi, a)-\phi_{B}$ is contained in $B\left(\delta_{10}\right)$, and $0<\left|p_{0} \psi-s\left(q_{0} \psi, a\right) \Phi_{0}\right| \leqslant 3 \eta(a) / 2$. We apply Proposition 13(i) and Proposition 12 and find $\left|q_{0} w_{T}-u\left(\left(\Psi_{0}, w_{T}\right)_{0}, a\right)\right| \leqslant k_{5}(3 \eta(a) / 2)^{u_{2} / u_{1}}$ for $T=$ $T(\psi, a)$ and for the solution $w=w(\psi, a):[-1, \infty) \rightarrow \mathbf{R}$ of $(a h)$ with $w_{0}=\psi$. Moreover,

$$
\begin{aligned}
P_{a} \phi-\bar{x}_{0}^{a} & =w_{T}+\phi_{A}-\bar{x}_{0}^{a}=p_{0} w_{T}-p_{0}\left(\bar{x}_{0}^{a}-\phi_{A}\right)+q_{0} w_{T}-q_{0}\left(\bar{x}_{0}^{a}-\phi_{A}\right) \\
& =p_{0}\left(P_{a} \phi-\phi_{A}\right)-p_{0}\left(\bar{x}_{0}^{a}-\phi_{A}\right)+q_{0} w_{T}-q_{0}\left(\bar{x}_{0}^{a}-\phi_{A}\right) \\
& =\delta_{7} \Phi_{0}-\delta_{7} \Phi_{0}+q_{0} w_{T}-u\left(\left(\Psi_{0}, \bar{x}_{0}^{a}-\phi_{A}\right)_{0}, a\right)
\end{aligned}
$$

(see Proposition 15(iii)).

With

$\left(\Psi_{0}, w_{T}\right)_{0} \Phi_{0}=p_{0} w_{T}=p_{0}\left(P_{a} \phi-\phi_{A}\right)=\delta_{7} \Phi_{0}=p_{0}\left(\bar{x}_{0}^{a}-\phi_{A}\right)=\left(\Psi_{0}, \bar{x}_{0}^{a}-\phi_{A}\right)_{0} \Phi_{0}$, we obtain

$$
\left|P_{a} \phi-\bar{x}_{0}^{a}\right|=\left|q_{0} w_{T}-u\left(\left(\Psi_{0}, w_{T}\right)_{0}, a\right)\right| \leqslant k_{5}(3 \eta(a) / 2)^{u_{2} / u_{1}} .
$$

(b) $P_{a}\left(D_{a}\right)$ is relatively compact: $P_{a}\left(D_{a}\right) \subset D_{a}$ is bounded, all $P_{a} \phi, \phi \in D_{a}$, are differentiable because of $T(\psi(\phi), a)+\tau \geqslant \tau \geqslant 1$, and the derivatives of the functions $P_{a} \phi$ are bounded by a $\max _{\mathbf{R}}|f|$.

(c) Schauder's fixed point theorem implies existence of a fixed point $\phi_{a}$ of $P_{a}$. For the solution $x:[-1, \infty) \rightarrow \mathbf{R}$ of equation $(a)$ with $x_{0}=\phi_{a}$, we have $x_{\tau+T(\psi, a)}-\phi_{B}$ $+\phi_{A}=\phi_{a}$, where $\psi:=x_{\tau}-\phi_{B}$, and it is easy to complete the proof.

REMARK ON BIFURCATION. Let $\varepsilon>0$ be given. Then one can choose $\hat{\delta}>0$ so small that for $a_{0}<a \leqslant a_{0}+\hat{\delta},\left|\bar{x}_{0}^{0}-\bar{x}_{0}^{a}\right| \leqslant \varepsilon / 2$ and $k_{5}(3 \eta(a) / 2)^{u_{2} / u_{1}}<\varepsilon / 2$. It follows that $\left|\phi_{a}-\bar{x}_{0}^{0}\right|<\varepsilon$ for every fixed point of $P_{a}$.

We collect results in

THEOREM 2. Let real numbers $A<0, B>0, r>0$ and a continuously differentiable function $\bar{f}: \mathbf{R} \rightarrow \mathbf{R}$ be given so that hypotheses $(\mathrm{H} 1)-(\mathrm{H} 6)$ are satisfied. Then there exists a parameter interval $\left[a_{0}, a_{2}\right) \neq \varnothing$, a continuous curve $\left[a_{0}, a_{2}\right) \ni a \rightarrow \bar{\phi}^{a} \in C$ of initial "alues, and a set valued mapping $\left(a_{0}, a_{2}\right) \ni a \rightarrow F_{a} \subset C, F_{a} \neq \varnothing$ for all $a \in\left(a_{0}, a_{2}\right)$, with the following properties.

(i) For each $a \in\left[a_{0}, a_{2}\right)$ there is a solution $\bar{x}^{a}: \mathbf{R} \rightarrow \mathbf{R}$ of equation

$$
\dot{x}(t)=a f(x(t-1)),
$$

where $f(\xi)=\bar{f}(\xi) / \bar{f}^{\prime}(A)$ for all $\xi \in \mathbf{R}$, so that $\bar{x}_{0}^{a}=\bar{\phi}^{a}$ and $\lim _{t \rightarrow-\infty} \bar{x}^{a}(t)=A$.

(ii) $\lim _{t \rightarrow+\infty} \bar{x}^{a_{0}}(t)=B$ and, for every $a \in\left(a_{0}, a_{2}\right), \bar{x}^{a}(t)$ does not converge to $B$ as $t$ tends to $+\infty$.

(iii) For every $a \in\left(a_{0}, a_{2}\right)$ and for every $\phi_{a} \in F_{a}$ there exists a periodic solution of the second kind $x(a): \mathbf{R} \rightarrow \mathbf{R}$ of equation $(a)$ with $x(a) \|[-1,0]=\phi_{a}$.

(iv) For every given neighbourhood $V$ of $\bar{x}_{0}^{a_{0}}$ there exists $\hat{\delta}>0$ such that $F_{a} \subset \bar{x}_{0}^{a_{0}}+V$ for $a_{0}<a<a_{0}+\hat{\delta}$. 
5. An example. Let $\rho \in(\pi / 2, \pi)$ be given. We set $\bar{f}_{\omega}(\xi):=\rho(\sin \omega-\sin (\xi+\omega))$ for $\xi \in \mathbf{R}$ and $\omega \in[0, \pi / 2)$. The equations (af ) with $a>0$ and $f:=\bar{f}_{\omega} / \bar{f}_{\omega}^{\prime}(\pi-2 \omega)$, $\omega \in[0, \pi / 2)$, model phase-locked loops for the control of high frequency generators (see e.g. [5]).

THEOREM 3. There exists $\omega_{0} \in(0, \pi / 2)$ such that for every $\omega \in\left(\omega_{0}, \pi / 2\right)$ the real numbers

$$
\begin{gathered}
A:=A_{\omega}:=-\pi-2 \omega, B:=B_{\omega}:=\pi-2 \omega, \\
r:=r_{\omega}:=B_{\omega} / 3=(\pi-2 \omega) / 3, \quad \xi_{1}:=\xi_{1, \omega}:=A_{\omega}+\omega, \quad \xi_{2}:=\xi_{2, \omega}:=-\omega
\end{gathered}
$$

and the function $\bar{f}$ satisfy

$$
A+2 r<\xi_{1}<\xi_{2}<-r<r<B-r
$$

and conditions $(\mathrm{H} 1)-(\mathrm{H} 6)$.

Proof. (*) holds true provided $2 \pi / 7<\omega<\pi / 2$, and (H1) and (H2) are clearly satisfied.

(a) There exists $\omega_{1} \in(2 \pi / 7, \pi / 2)$ such that for every $\omega \in\left(\omega_{1}, \pi / 2\right),-r_{\omega} / 2<\bar{f}_{\omega}$ $<r_{\omega} / 2$ in $\left(A_{\omega}-r_{\omega}, A_{\omega}+r_{\omega}\right) \cup\left(-r_{\omega}, B_{\omega}-r_{\omega}\right.$. Proof: It is enough to show that $-r_{\omega} / 2<\min \bar{f}_{\omega}=\bar{f}_{\omega}((\pi / 2)-\omega)$ and that $\bar{f}_{\omega}\left(B_{\omega}+r_{\omega}\right)<r_{\omega} / 2$ for $\omega$ sufficiently close to $\pi / 2$. Set $G_{1}(\omega):=\bar{f}_{\omega}((\pi / 2)-\omega)=\rho(\sin \omega-1), H_{1}(\omega):=-r_{\omega} / 2=$ $-(\pi-2 \omega) / 6, \quad G_{2}(\omega):=\bar{f}_{\omega}\left(B_{\omega}+r_{\omega}\right)=\rho(\sin \omega-\sin ((4(\pi-2 \omega) / 3)+\omega))$, $H_{2}(\omega):=r_{\omega} / 2=(\pi-2 \omega) / 6$ for all $\omega \in \mathbf{R}$. Then $G_{j}(\pi / 2)=0=H_{j}(\pi / 2)$ and $G_{j}^{\prime}(\pi / 2)=0$ for $j \in\{1,2\}, H_{1}^{\prime}(\pi / 2)=1 / 3, H_{2}^{\prime}(\pi / 2)=-1 / 3$. This implies the assertion.

(b) There exists $\omega_{2} \in\left(\omega_{1}, \pi / 2\right)$ such that for every $\omega \in\left(\omega_{2}, \pi / 2\right), \bar{f}_{\omega}^{\prime}<1$ in $\left[A_{\omega}-r_{\omega}, A_{\omega}+r_{\omega}\right]$. Proof: Choose $\omega_{2}^{\prime} \in\left(\omega_{1}, \pi / 2\right)$ with

$$
B_{\omega}+r_{\omega}=4(\pi-2 \omega) / 3<(\pi / 2)+(\pi-2 \omega) / 2=B_{\omega} / 2+\pi / 2
$$

for all $\omega \in\left(\omega_{2}^{\prime}, \pi / 2\right)$. Then $\bar{f}_{\omega}^{\prime}$ is nonnegative and increasing on $\left[B_{\omega}-r_{\omega}, B_{\omega}+r_{\omega}\right]$. It remains to show that for $\omega$ sufficiently close to $\pi / 2,1>\bar{f}_{\omega}^{\prime}\left(B_{\omega}+r_{\omega}\right)=$ $-\rho \cos ((4(\pi-2 \omega) / 3)+\omega)$, which is obvious.

(c) There exists $\omega_{3} \in\left(\omega_{2}, \pi / 2\right)$ such that for every $\omega \in\left(\omega_{3}, \pi / 2\right), a_{\omega}^{+}:=\bar{f}_{\omega}^{\prime}\left(A_{\omega}\right)$ satisfies $\log a_{\omega}^{+}<-u\left(a_{\omega}^{+}\right)$. This follows from $\bar{f}_{\omega}^{\prime}\left(A_{\omega}\right)=-\rho \cos (-\pi-\omega)$, from $\lim _{a \rightarrow 0+} \log a=-\infty$ and from $\lim _{a \rightarrow 0+} u(a)=0$.

(d) There exists $\omega_{4} \in\left(\omega_{3}, \pi / 2\right)$ such that for every $\omega \in\left(\omega_{4}, \pi / 2\right)$ we have $\xi_{1, \omega}+\int \bar{f}_{\omega} \circ \phi \leqslant \xi_{2, \omega}$ provided $\phi \in C, A_{\omega} \leqslant \phi \leqslant \xi_{1, \omega}=\phi(0)$, and $B_{\omega}+r_{\omega}<\xi_{2, \omega}+$ $\int \bar{f}_{\omega} \circ \psi$ provided $\psi \in C, \xi_{1, \omega} \leqslant \psi \leqslant \xi_{2, \omega}=\psi(0)$. Proof: Let $\omega \in\left(\omega_{3}, \pi / 2\right)$. For every $\phi \in C$ with $A_{\omega} \leqslant \phi \leqslant \xi_{1, \omega}=\phi(0)$ we obtain

$$
\begin{aligned}
\xi_{1, \omega}+\int \bar{f}_{\omega} \circ \phi & \leqslant A_{\omega}+\omega+\bar{f}_{\omega}\left(A_{\omega}+\omega\right) \\
& =-\pi-\omega+\rho(\sin \omega-\sin (-\pi)) \leqslant-\omega=\xi_{2, \omega}
\end{aligned}
$$

because of $\rho<\pi$. Furthermore,

$$
\xi_{2, \omega}+\int \bar{f}_{\omega} \circ \psi \geqslant-\omega+\bar{f}_{\omega}(-\omega)=-\omega+\rho(\sin \omega-\sin (0))=-\omega+\rho \sin \omega
$$




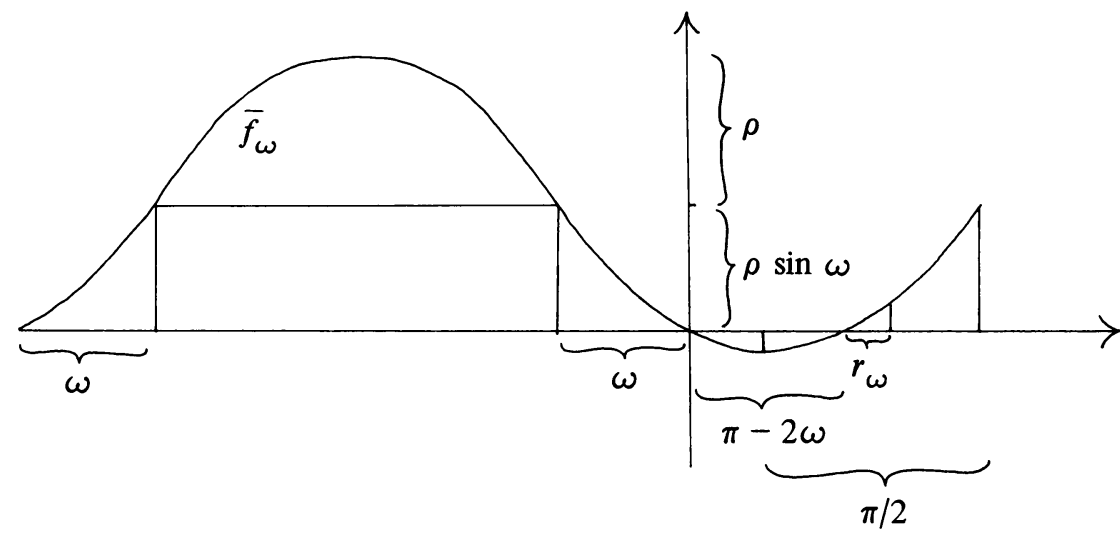

Figure 6

in case $\xi_{1, \omega} \leqslant \psi \leqslant \xi_{2, \omega}=\psi(0), \quad \psi \in C$. Recall $B_{\omega}+r_{\omega}=4(\pi-2 \omega) / 3$. Set $G_{3}(\omega):=-\omega+\rho \sin \omega, H_{3}(\omega):=4(\pi-2 \omega) / 3$ for $\omega \in \mathbf{R}$. Then $G_{3}(\pi / 2)=-(\pi / 2)$ $+\rho>0=H_{3}(\pi / 2)$, and the desired estimate follows by continuity for $\omega$ sufficiently close to $\pi / 2$.

(e) Let $\omega \in\left(\omega_{4}, \pi / 2\right)$. (a), (b), (c), (d) imply (H3), (H4), (H5), (H6), respectively.

\section{REFERENCES}

1. A. A. Andronov and C. E. Chaikin, Theory of oscillations, Princeton Univ. Press, Princeton, N.J., 1949.

2. N. Chafee, A bifurcation problem for a functional differential equation of finitely retarded type, J. Math. Anal. Appl. 35 (1971), 312-348.

3. S. N. Chow and J. K. Hale, Methods of bifurcation theory, Springer, New York, Heidelberg and Berlin, 1982.

4. S. N. Chow and J. Mallet-Paret, Singularly perturbed delay-differential equations, Coupled Nonlinear Oscillators (J. Chandra and A. C. Scott, eds.), North-Holland Math. Studies, vol. 80, 1983, pp. 7-12.

5. T. Furumochi, Existence of periodic solutions of one-dimensional differential-delay equations, Tôhoku Math. J. 30 (1978), 13-35.

6. J. K. Hale, Functional differential equations, Springer, New York, Heidelberg and Berlin, 1971.

7. __ Theory of functional differential equations, Springer, New York, Heidelberg and Berlin, 1977.

8. J. K. Hale and K. Rybakowski, On a gradient-like integro-differential equation, Proc. Roy. Soc. Edinburgh Sect. A 92 (1982), 77-85.

9. J. Mallet-Paret and R. D. Nussbaum, Global continuation and asymptotic behavior of periodic solutions of differential-delay equations, in preparation.

10. L. P. Sil'nikov, On the generation of periodic motion from trajectories doubly asymptotic to an equilibrium state of saddle type, Math. USSR-Sb. 6 (1968); English transl., Mat. Sb. 77(119) (1968), 427-438.

Mathematisches Institut der Universität MÚNChen, D 8000 MÚNChEN 2, West Germany 